\title{
A Computational Study of Intradural Spinal Cord Stimulation
}

\author{
A Thesis \\ Presented to \\ the faculty of the School of Engineering and Applied Science \\ University of Virginia \\ In Partial Fulfillment \\ of the requirements for the Degree \\ Master of Science
}

By

Qiujun Huang

December, 2012 


\section{APPROVAL SHEET}

This thesis is submitted in partial fulfillment of the requirements for the degree of

Master of Science

Qiujun Huang

Approved:

Marcel. Utz (Advisor)

George T. Gillies (Chair)

Michael L. Reed

Accepted by the School of Engineering and Applied Science:

James H. Aylor (Dean)

December, 2012 


\section{Abstract}

Spinal Cord Stimulation (SCS) is a modality used by many clinicians to treat medically refractory pain and several other chronic conditions. However, standard epidural approaches to SCS are subject to a number of performance limitations that can produce adverse side effects and result in modest, if any, therapeutic effect in many patients.

Intradural SCS (ISCS) is being investigated as a possible new method of neuromodulation aimed at overcoming these problems. By direct stimulation of the pial surface, we seek to obtain more focused excitation of the targeted fibers in the spinal cord, while avoiding inadvertent activation of the neighboring non-targeted ones.

Recent experimental studies at the University of Iowa and the theoretical modeling work at the University of Virginia have laid the groundwork for a greater efficacy and better safety through ISCS. A three-dimensional (3-D) model of SCS has been developed to explore the therapeutic mechanism underlying the approach. In partic-

ular, computational results allow comparison between the epidural SCS (ESCS) and ISCS modalities and demonstrate improved stimulation efficacy and accuracy from ISCS. 


\section{Acknowledgements}

I would like to thank my advisor Professor Marcel Utz for his guidance and support through out this research. I also would like to express my gratitude toward Professor George T. Gillies for his valuable suggestions and help.

Many thanks to colleagues at the University of Iowa including Professor Matthew A. Howard III, Professor Hiroyuki Oya and Dr. Oliver E. Flouty for their cooperation and assistance.

Most importantly, thanks to my parents and friends for unconditional support throughout my education. 


\section{Contents}

List of Figures $\quad$ v

List of Tables $\quad$ X

1 Introductions 1

1.1 Introduction to Spinal Cord Stimulation . . . . . . . . . . . . . . 1

1.2 Introduction to Intradural Spinal Cord Stimulation . . . . . . . . . . 2

1.3 Organization of the Thesis . . . . . . . . . . . . . 3

2 Literature Review 5

2.1 Exciation of Nerve Fibers . . . . . . . . . . . . . . . . 5

2.2 Evolution of ESCS . . . . . . . . . . . . . . . . . 7

2.3 Limitations of $\mathrm{ESCS} \ldots \ldots \ldots \ldots$

3 Materials and Methods $\quad 12$

3.1 Experimental Model . . . . . . . . . . . . . . . . . . 12

3.2 Computational Model . . . . . . . . . . . . . . 16

3.2 .1 Volume Conductor Model . . . . . . . . . . . . . . . . . 16

3.2.2 Nerve Fiber Model . . . . . . . . . . . . . . . . . . . . . . . . 20

4 Results and Discussion $\quad 29$ 
4.1 Current Source Stimulation _. . . . . . . . . . . . . . . . 29

4.1.1 Electrical Potential Field in the Spinal Cord . . . . . . . . . . 30

4.1.2 Membrane Depolarization Voltage Distribution . . . . . . . . 32

4.2 Voltage Source Stimulation . . . . . . . . . . . . . . . 43

4.2.1 Electrical Potential Field in the Spinal Cord Generated by Voltage Source Stimulation . . . . . . . . . . . . . . . . . 43

4.2.2 Membrane Depolarization Voltage Distribution . . . . . . . . 45

4.3 Comparison between Modeling and Experimental Results . . . . . . . 54

4.4 Conclusion . . . . . . . . . . . . . . . . . . . . . . . . 59

5 Future Scope and Directions $\quad 61$

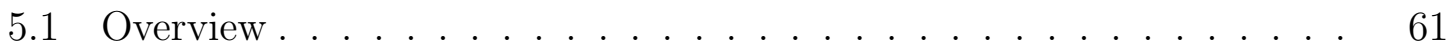

5.2 Future Work . . . . . . . . . . . . . . . . . 62

$\begin{array}{ll}\text { Bibliography } & 63\end{array}$ 


\section{List of Figures}

3.1 The experimental arrangement for electrical stimulation of the dorsal columns in the ovine spinal cord is shown here, with (a) a sheep diagram showing the position of electrode on spinal cord, and (b) a cross-sectional view of the spinal cord and surgical set-up. . . . . .

3.2 The stimulation pulse applied on cathode. The pulse has two phases of $20 \mu$ s positive rectangular wave and $1411 \mu$ s negative rectangular wave(partially shown here). The negative wave amplitude is $1.4 \%$ of the positive wave amplitude to maintain charge balance on electrodes surfaces. . . . . . . . . . . . . . . . .

3.3 The three dimensional (a)anatomical structure (Blue: CSF; Red: White Matter; Grey: Grey Matter.) and (b)mesh configuration of spinal cord. The model has $20 \mathrm{~mm}$ length in vertical direction and about $10 \mathrm{~mm}$ transverse diameter. Physics controlled mesh setting is utilized here.

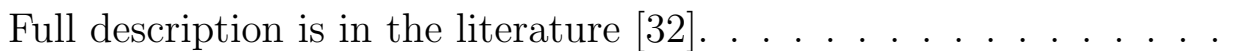

3.4 The circuit model used for fiber segmentation [25]. Subscripts $e$ and $j$ represent the external voltage at fiber node $j . \ldots . . . . . .$. 
4.1 The potential field on the surface of the spinal cord for both case of the (a) ESCS with $10 \mathrm{~mA}$ stimulations and the (b) ISCS with $1 \mathrm{~mA}$ stumulation; the color represents the amplitude of the depolarization voltage. . . . . . . . . . . . . . . . . . . . 31

4.2 Continuing. . . . . . . . . . . . . . . . . 34

4.2 The depolarization voltage on cross sections of (a) $0.5 \mathrm{~mm}$ above the cathode (b) on the same level as the cathode (c) $0.5 \mathrm{~mm}$ below the cathode; the color represents the amplitude of voltage in units of volts.

4.3 Continuing . . . . . . . . . . . . . . . . . . . . 36

4.3 The depolarization voltage on cross sections at (a) $0.5 \mathrm{~mm}$ above the cathode (b) on the same level as the cathode (c) $0.5 \mathrm{~mm}$ below the cathode; the color represents the amplitude of voltage in units of volts.

4.4 The depolarization on each axonal node of the target nerve fiber for the case of ESCS and ISCS. Red: ESCS; Blue: ISCS. . . . . . . . . . 38

4.5 The depolarization voltage within cross sections on the same level as the cathode: (a) ESCS with $10 \mathrm{~mA}$ stimulation and (b) ISCS with 1 mA stimulation; the color represents the amplitude of depolarization voltage. . . . . . . . . . . . . . . . . .

4.6 The depolarization voltage of (a) ESCS and (b) ISCS; the color represents the amplitude of voltage. Cross sections parallel to the $Y-Z$ plane and through the center of the spinal cord. . . . . . . . . .

4.7 The potential field on the surface of spinal cord for both case of (a) ESCS and (b) ISCS; the color represents the amplitude of voltage in the units of volts. . . . . . . . . . . . . . . . . . . 44

4.8 Continuing . . . . . . . . . . . . . . . . . . . 46 
4.8 The depolarization voltage on cross sections at (a) $0.5 \mathrm{~mm}$ above cathode (b) on the same level at the cathode (c) $0.5 \mathrm{~mm}$ below the cathode; the color represents the amplitude of voltage in units of volts. . . . . 47

4.9 Continuing . . . . . . . . . . . . . . . . . . . . 48

4.9 The depolarization voltage on cross sections (a) $0.5 \mathrm{~mm}$ above the cathode (b) on the same level as the cathode (c) $0.5 \mathrm{~mm}$ below the cathode; the color represents the amplitude of voltage in units of volts.

4.10 The depolarization voltage color contours of (a) ESCS and (b) ISCS; Cross sections parallel to the $X-Y$ plane are on the same level as the cathode. The legend shows the color code in the range between -0.05 $\mathrm{V}$ and $0.05 \mathrm{~V} \ldots \ldots \ldots \ldots \ldots \ldots$

4.11 The depolarization voltage of (a) ESCS and (b) ISCS; the color represents the amplitude of voltage. Cross sections parallel to the $Y-Z$ plane are through the center of the spinal cord. . . . . . . . . . .

4.12 Red color shows excited area on the spinal cord under the condition of a $1 \mathrm{~V}$ applied voltage difference on the electrodes (a)ESCS has a wider and thinner excitation area than (b)ISCS. Because the Gracile fasciculus is located on the spinal cord from the medial to the lateral part, a focused excitation is essential to void side effects. . . . . . . . 53

4.13 The blue area represents the horizontal cross section of the spinal cord; the red square is the excitation target with $0.5 \mathrm{~mm}$ side length. . . . 55

4.14 Continuing . . . . . . . . . . . . . . . . . . . 57 
4.14 With different applied voltages, the (a)experimental result shows the observed ERP at the brain channel with maximum response, and the associated fitted curves; while the (b) modelling result demonstrates the total maximum depolarization voltages of excited fibers in the target zone and the fitted curve. Red and blue colors here represent ISCS and ESCS respectively. . . . . . . . . . . . . . . . . . 58 


\section{List of Tables}

3.1 Material Conductivity . . . . . . . . . . . . . . . 20

3.2 Physiological Parameters of Never Fibers . . . . . . . . . . . . . 24 


\section{Chapter 1}

\section{Introductions}

\subsection{Introduction to Spinal Cord Stimulation}

In 1965, Melzack and Wall published the gate control theory of pain in Science [1]. That theory proposes that there is a neurophysiological gate in the dorsal horn of spinal cord, which governs the transmission of pain signal in neuron activities. The "gate" could be closed by stimulating large afferents in it, thereby blocking or reducing pain signal transmission between the spinal cord and brain.

Based on the gate theory, Spinal Cord Stimulation (SCS) was first used in 1967 to treat intractable pain in cancer patients [2]. Since then the clinical applications for SCS and the devices themselves have evolved significantly [3], and this modality is now used to treat roughly 35,000 patients per year in the U.S. [4]. In the past 60 years of research on SCS, computational modeling has provided a solid empirical basis for clinical applications, and helped achieve optimal design of SCS devices [5, 6], improve the properties of applied stimulation $[7,8]$ and the control of side effects $[9,10]$.

However, it remains difficult for standard epidural SCS (ESCS) to achieve a balance between therapeutic efficacy and functional reliability: ESCS fails over the long 
term in up to half of all patients with these implants [11]. This is in part because the therapeutic window for consistent and effective treatment is very narrow: to reach the stimulation threshold for any fibers other than those in a thin band about 300 $\mu \mathrm{m}$ deep around the spinal cord periphery, signal strengths beyond the threshold for discomfort are typically needed. Shunting effects by the relatively high conductivity cerebrospinal fluid (CSF), shifts in lead position and other systematic effects underlie this inability produce a more robust therapeutic window of operation. Therefore, attempts to localize the stimulus pattern accurately within the targeted fiber regions typically result in current density leakage into neighboring untargeted structures with concomitant production of paresthesias and other painful sensations, eg., due to activation of the dorsal root entry zones.

\subsection{Introduction to Intradural Spinal Cord Stim- ulation}

To overcome these limitations of ESCS, our collaboration is developing a novel approach to SCS that places the electrode array directly on the pial surface of the spinal cord [12]. This intradural approach to spinal cord stimulation (ISCS) holds promise of being a more focused form of neuromodulation than ESCS, thus potentially offering a wider therapeutic window and careful targeting of specific fiber bundles within the dorsal columns. Our initial goal is to develop this modality for the treatment of medically refractory pain, but future applications may include movement disorders associated with neurodegenerative diseases and spinal cord injury.

In this thesis, experimental observations made during a recent series of in vivo ISCS studies on a large animal model $[13,14,15]$ are briefly introduced. Thereafter, a 3-D computational model for SCS and the results of its use to predict the changes 
in 3-D depolarization voltage distributions within the spinal cord are presented and discussed.

Experimental observations and modeling predictions reach agreement that with the same input stimulation ISCS improves upon ESCS in both efficacy and safety. The 3-D model developed here enables systematic study of the parameters involved, including electrodes configurations, nerve fiber geometry and so forth. The experimental results represent systemic nerve response functions and our 3-D model seeks provide insight into the biophysical properties of nerve fibers and spinal cord tissues and shed some light on the mechanism of SCS.

\subsection{Organization of the Thesis}

The thesis is organized as following:

In chapter 2, a series of papers are reviewed to provide background information on nerve fiber stimulation as well as the initiation and development of ESCS. The current status and limitation of ESCS is discussed.

In chapter 3, a series of experiments on animal subjects is introduced. Then, the computational method and model that is compared with the experimental set-up is illustrated. By using the FEM and nerve fiber circuit model, the computational calculation determines the electrical potential field on the spinal cord and simulates the initiation of transmission potential in the nerve fibers.

In chapter 4, computational result of ISCS and ESCS are compared to demonstrate the improved efficacy and safety achieved by ISCS. Based on the modeling results, a theoretical model is proposed to explain the experimental results. This theoretical model is also used to investigate the mechanism of SCS.

In chapter 5 , the properties of the ISCS are summarized and the computational 
model is developed. The significance of ISCS and future applications of the computational model are also discussed. 


\section{Chapter 2}

\section{Literature Review}

In this chapter, I will give a brief review to the initiation and evolution of the ESCS. Focus is given to significant works in the history of SCS development, which lead to the current standard methodology of SCS.

\subsection{Exciation of Nerve Fibers}

Determining the excitation status is essential to SCS. The study of nerve fiber excitation is closely related but not restricted to the development of ESCS. Therefore, before reviewing the evolution of SCS, I will give a brief introduction to the studies of nerve fiber excitation.

The first mathematical model, which successfully described the conduction and excitation in nerve fibers, was proposed by Hodgkin and Huxley (H-H equations) in the 1950s [16]. This theory began to reveal the mechanism of nerve excitation and signal transmission. The $\mathrm{H}-\mathrm{H}$ equations state that the electrical behavior of nerve membranes can be represented by an electrical circuit. The components and electrical properties depend on the extracellular potential and the physiologic properties of the 
nerve. Thus, membrane potentials of a nerve fiber can be determined by solving a series of differential equations which govern the electrical circuit. In the 1960s, Frankenhaeuser and Huxley empirically modified H-H equations for myelinated frog nerve, and those results are now widely accepted as the Frankenhaeuser-Huxley (F-H) equations[17].

In 1976, McNeal summarized previous work on nerve fiber excitation and established a model for excitation of myelinated nerve [18]. This model allowed computations with an excitation pulse of aritrary duration and electrode configurations. Moreover, this model provided for the calculation of time-dependent membrane potentials at each of the nodes of Ranvier up to the time of action potential initiation. McNeal's model is widely accepted in various neuroelectric stimulations to predict the initiation of the action potential.

Based on McNeal's model, two major methods were developed to determine the status of nerve fibers under an applied electric field. For the first method, Sweeney et al. studied the subthreshold behavior of nerve fibers, in which the membrane conductance is constant [19]. In this method, the excitation threshold for the membrane voltage was assumed to be an constant under all conditions. However, this threshold on membrane conductance was later shown to depend on applied pulse width by Warman [8]. The second method developed by Rattay used the second spatial derivative of the external electrical field along the path of a nerve fiber to give the most likely site of action potential initiation on the nerve fiber [20,21]. Because Ratty's model did not include physiological information for the nerve fiber, it cannot reflect the complicated reaction of the nerve fiber under excitation.

In 1992, Warman et al. published a new model combining the works of McNeal and Ratty. This model included the complexity of the neuron physiological properties and the variances of the external potential field distributions [8]. More importantly, this 
model defined a critical transmembrane potential as the reference for determining the initiation of axonal node excitation. Moreover, the influence of pulse width, electrode configuration, and nerve fiber geometry were demonstrated. This work provided a practicable standard for determining the anode excitation in a complex neural system with numerous nerves.

\section{$2.2 \quad$ Evolution of ESCS}

The gate theory of pain proposed by Melzak and Wall [1] is often used as the starting point for investigating possible mechanisms of action for spinal cord stimulation. In 1967, the first case of spinal cord stimulation for pain control was carried out by Shealy et al [2]. After that, ESCS was increasingly applied in various pain treatments, such as chronic pain syndrome [22], motor disorders [23], Parkinson disease [24] and so forth.

The development of ESCS largely depends the progress in the understanding of neuronal excitation mechanisms. Hodgkin and Huxley's mathematical model of nerve fibers brought insights to the understanding of ESCS mechanism. Based on the $\mathrm{H}-\mathrm{H}$ equation, the F-H equation and the subsequent McNeal model, computational modelling has been employed in the study of ESCS for more than 30 years $[16,17,18]$. The development of ESCS modelling shred lights on ESCS mechanisms, provided predictions for ESCS clinical applications, and also induced continuous effort on refinement of the ESCS models themselves.

In 1980, an ESCS computational model was introduced by Coburn [25]. In this work, Coburn used a two-step method to calculation the effect of ESCS. First, the electrical potential field on the spinal cord is calculated by Finite Elemental Methods (FEM). Second, the nerve fiber model was used to calculate the depolarization volt- 
ages on nerve fibre nodes, in which the electrical potential field obtained from the first step served as the boundary condition. This two-step framework has been inherited by later studies in ESCS. However, Coburn assumed that the depolarization voltages on nerve fiber nodes have a linear relation with the external electrical potential field, which neglects the complexity of the nerve fibers. This assumption significantly decreased the computation intensity, but also raised many questions about the accuracy of results.

The computational model was significantly furthered by scholars at the University of Twente. Struijk and Holsheimer et al. undertook a deep investigation of the excitation of dorsal column fibers $[26,27]$. They used a computational model to predict the influence of electrode configuration and neuronal anatomical parameters. They tried different positions and configurations of electrodes. They demonstrated that fiber diameter, electrode-fiber distance, and electrode-fiber orientation are significant in selective fiber stimulation.

Because the dorsal root (DR) fibers also play an important role in ESCS, Struijk and Holsheimer et al. also conducted a theoretical study of DR fiber excitation [28]. They found that DR fibers with different curvature respond differently to a given external potential field. Also increasing the width of the CSF layer caused a decrease in the average depolarization voltages in the axonal nodes. Similar to the excitation of DC fibers, this illustrated that the position and configuration of stimulating electrodes has a direct and significant impact on the excitation result.

Among all the parameter tested such as fiber diameter, fiber curvature and CSF layer width etc., the configuration of electrodes is the only controllable parameter to improve the effectiveness of ESCS in application. Thus many efforts were undertaken to study the function of ESCS with various electrode configurations [5, 6, 29]. In 1997, Holsheimer and Wesselink optimized the geometry of electrodes to further decrease the 
stimulation threshold [30]. They used longitudinal electrodes arrays in the epidural space. Two types of electrodes were studied: general percutaneous ones and those requiring a laminectomy. Each individual electrode is rectangular, with the shape defined by contact length (parallel to longitudinal direction) and contact width. The gap between electrodes was defined as the contact separation. With this investigation, they found several limitations on the design of ESCS electrode configurations. Large current density at the contact-tissue interface may cause harmful electrochemical reactions in the contacted tissue. Therefore, there is a low boundary for the size of electrodes length, width and separation. Moreover, because the width of the CSF layer varies along the spine and between subjects, it is impossible to find a universal optimum electrode geometry. Hence their result demonstrates optimum electrode geometry corresponding only to certain CSF layer widths.

With the development of SCS, safety issues have increasingly become ever more important. In Cameron's report [10], the effectiveness and safety of SCS was studied based on clinical data from 3679 patients. A successful treatment patient was defined as one who has greater than $50 \%$ pain relief. With this modest pain relief standard, this report discussed complications associated with this clinical application. Lead migration and breakage, unwanted stimulation as well as battery failure are a major categories of complications.

\subsection{Limitations of ESCS}

As mentioned above, the ESCS has been under development for more than 60 years with wide clinical application. But several critical issues remain unsolved.

The ideal outcome of ESCS in clinical application is that the patient obtains $100 \%$ pain relief without any side effects. However, feedback from clinical observations [10] 
demonstrated that ESCS can only achieve modest pain relief at best, and also cause undesired stimulations. According to Cameron's work, a successful treatment via ESCS is defined as 50\% pain relief. Even with this plain standard, the success rate of ESCS treatments for low-back and leg pain, ischemic limb pain as well as complex regional pain syndrome are $62 \%, 77 \%$, and $84 \%$ respectively. Because of the inherent limitations, it is difficult for ESCS to achieve a higher level of efficacy and safety in clinical application. The reason is that, in ESCS, the stimulation electrodes are always separated from the white matter by the CSF, which has much a higher electric conductivity than the other tissues in the spinal cord. Thus, the CSF layer absorbs most of the stimulation energy and makes the electric potential field of less intensity inside the white matter. Increasing the stimulation intensity at the electrodes could enlarge the stimulation effectiveness on the target nerves, but the price is that more energy is conducted to nearby body tissues and unwanted nerves could also be stimulated. This paradox makes ESCS fail to balance effectiveness and safety. The original motivation for ISCS is to solve this problem by implanting electrodes under the CSF layer in direct contact with white matter. Further, the width of the CSF layer varies among patients and this width has a large impact on the stimulation effectiveness. Thus, ESCS computational results depend strongly on CSF width, and it is difficult to predict treatment outcome for a given patient. In contrast, ISCS has no such issue at all.

Moreover, the mechanism of SCS is still obscure. Technically, the effectiveness of SCS is based on the statistic data of clinical outcomes. The excitation status changes of neural nodes on spinal cord caused by stimulation is provided by computational modelling. It has been a challenge to connect the stimulation result on the spinal cord with pain relief outcomes in clinical trials. In studies of ESCS, most attention is paid to the binary excitation status of a single spinal cord fiber. In this thesis, a theory 
is proposed to include the stimulation intensity of a cluster of fibers in a certain area on spinal cord, which allows for direct comparison between modeling results on the spinal cord and the associated experimental results. 


\section{Chapter 3}

\section{Materials and Methods}

In this chapter, I will briefly describe a series of experimental studies done by our collaborators at the University of Iowa. This is an adjunct to the main focus of this thesis, which is on theoretical methods, which are discussed in detail later, along with a comparison between the theoretical results and experimental results.

\subsection{Experimental Model}

Laboratory tests of ISCS vs. ESCS were carried out as part of an institutionally approved series of in vivo ovine studies (4 trials) done at the University of Iowa (Fig. 3.1) $[13,14,15]$. The stimulator used in those experiments consisted of a probe positioned by a micromanipulator, with a pair of electrodes at the probe's distal tip. Each electrode was a $0.5 \mathrm{~mm}$ radius hemisphere, and they were separated edge-to-edge by $1 \mathrm{~mm}$ of insulation. The orientation of the electrode pair was along the rostralcaudal axis of the spinal cord, and the location of the probe relative to the dorsal midline of the spinal cord is shown in the top and cross-sectional views presented in Fig. 3.3. The input signals were constant voltage, charge-balanced, mono-phasic 
square pulses of $20 \mu$ s positive duration and $1411 \mu$ s negative duration (Fig. 3.2). 


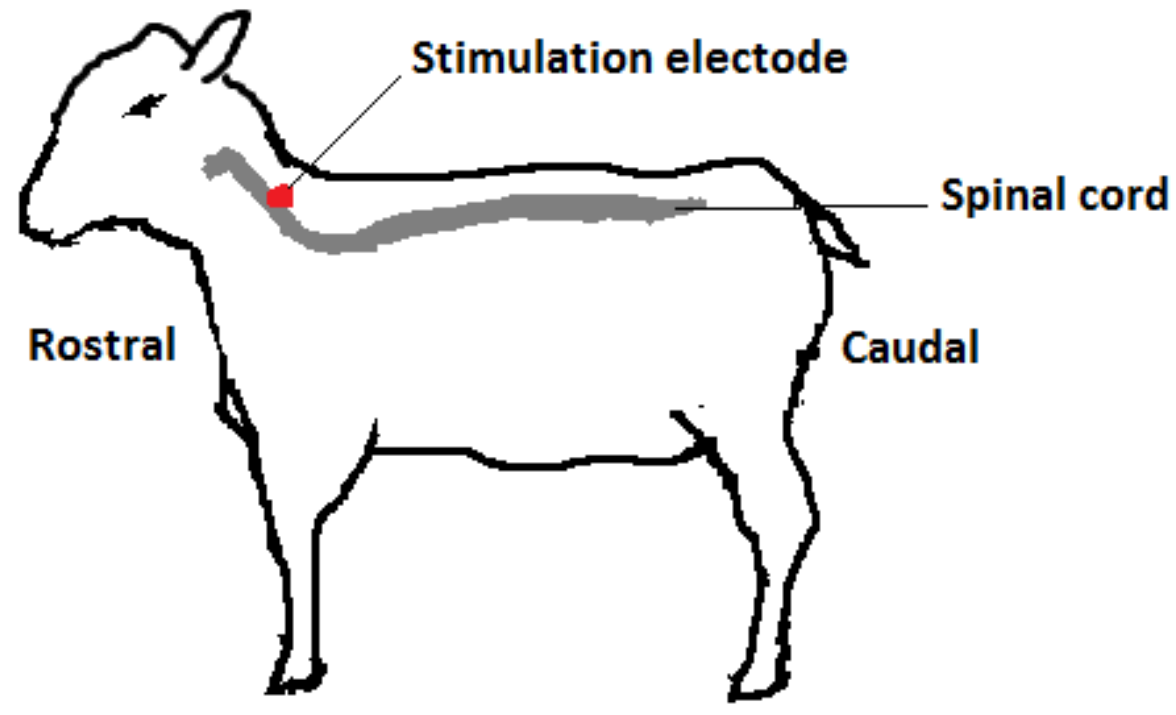

(a) sheep surgery diagram

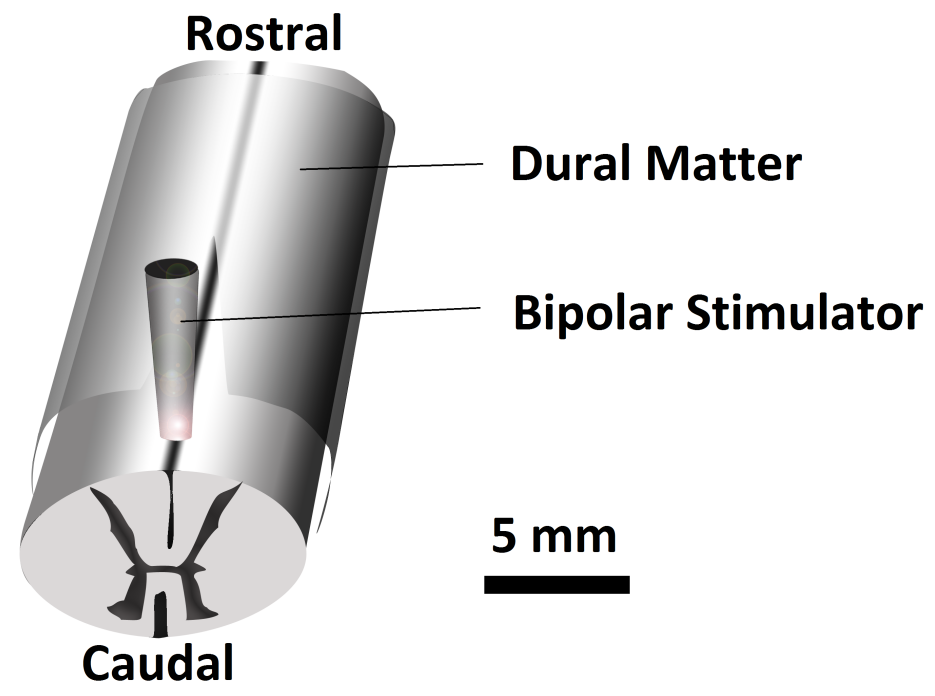

(b) schematical surgery set-up

Figure 3.1: The experimental arrangement for electrical stimulation of the dorsal columns in the ovine spinal cord is shown here, with (a) a sheep diagram showing the position of electrode on spinal cord, and (b) a cross-sectional view of the spinal cord and surgical set-up. 


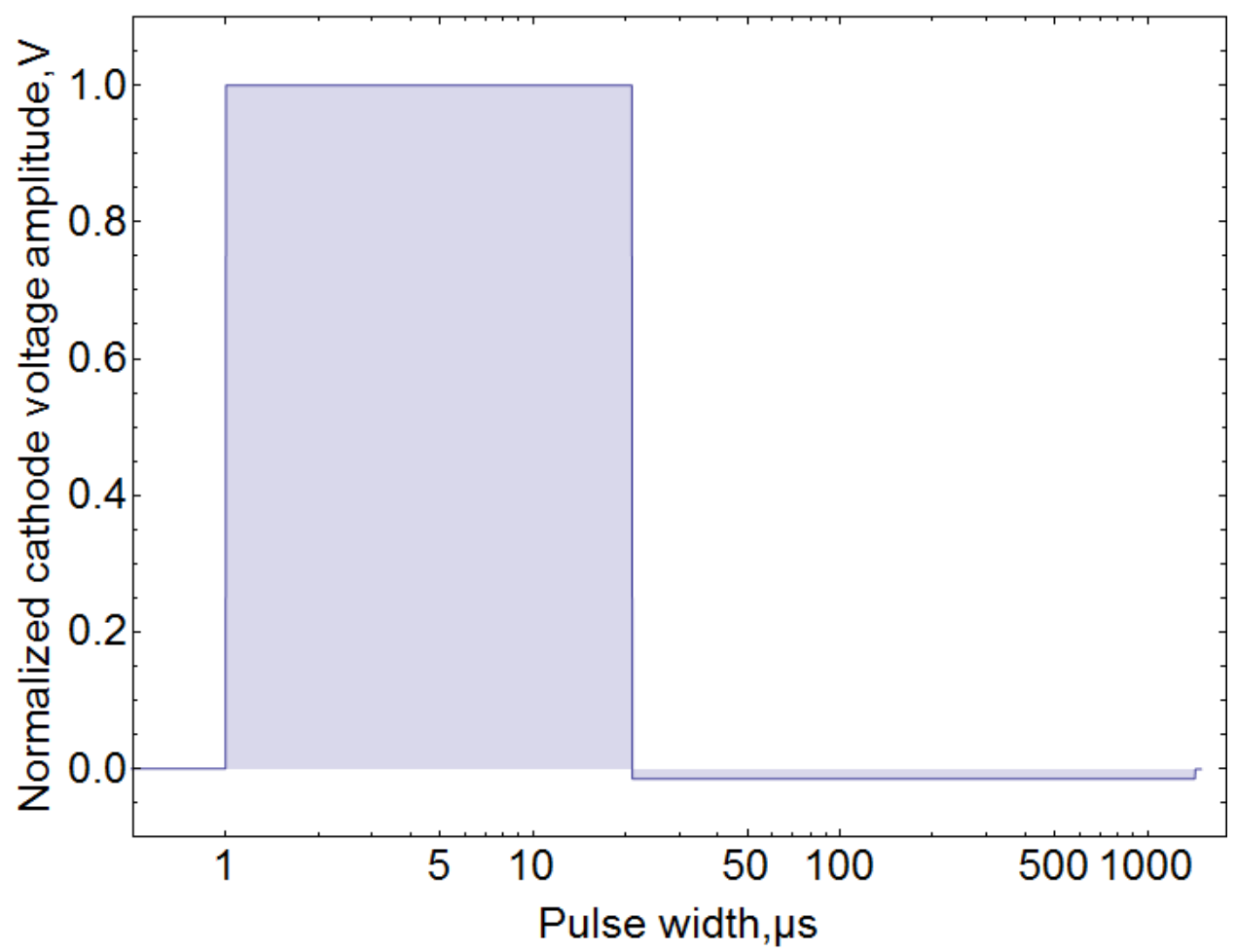

Figure 3.2: The stimulation pulse applied on cathode. The pulse has two phases of $20 \mu$ s positive rectangular wave and $1411 \mu$ s negative rectangular wave(partially shown here). The negative wave amplitude is $1.4 \%$ of the positive wave amplitude to maintain charge balance on electrodes surfaces. 
To characterize the nature of the cortical response to intradural vs. epidural SCS, right-sided craniectomy was carried out and a multi-electrode (60 channel) array with $3 \mathrm{~mm}$ inter-contact spacing (Ad-Tech Medical Instruments, Racine, Wisconsin, USA) was placed on the surface of the exposed brain. The signals from each channel were recorded as the stimuli were presented at different amplitudes and distances from the surface of the spinal cord (ISCS) and the top of the CSF layer (ESCS), respectively, with and without tibial nerve stimulation. The resulting data were filtered and analyzed and, in the next chapter Fig. 4.13(a) shows representative findings from one of the several channels of the array that exhibited a clear difference in the magnitude and onset of the evoked responses produced by ISCS vs. ESCS as a function of the stimulus voltage.

\subsection{Computational Model}

Accurate modeling of neuromodulation in the spinal cord involves analysis of both overall dorsal column stimulation and nerve fibers stimulation. Regarding the former, a three-dimensional Finite Element Model (FEM) based on the anatomical structure and Electrical properties of the spinal cord is used to calculate the potential gradient on the spinal cord. Then, the nerve fiber model utilizes this result as a boundary condition to determine the depolarization inside each neuronal node.

\subsubsection{Volume Conductor Model}

It is routine to calculate the voltage distribution on the spinal cord by using FEM volume conductor models $[31,26,27]$. We employed the Comsol 4.2a Multiphysics Package [32] to synthesize a FEM-based 3-D volume conductor representation of the anatomical structure of the spinal cord (Fig. 3.2) which incorporates the conductivi- 
ties of the relevant tissues, as listed in Table 3.1.

Although the anatomical structure of the spinal cord changes along its axial direction, the difference is not significant in terms of electrical properties a few centimetres in range. Thus a widely adopted simplification is that focusing on the more complicated transverse structure, and ignoring the vertical variance [31, 26, 27]. Our 3-D model is extruded from the same transverse geometry which is based on that of the ovine spinal cord as determined by MR imaging (Fig. 3.2). The length of the spinal cord segment within our computational model is $20 \mathrm{~mm}$, with periodic boundary conditions applied at both ends. The model length is long enough for the electrical field generated by $1 \mathrm{~mm}$ diameter electrodes to decay completely, while meanwhile reaching a balance with computational efficiency. Bipolar stimulation is assumed in the model, with the electrode pair positioned along the longitudinal direction of spinal cord. The electrodes are $0.5 \mathrm{~mm}$ in radius with a $1 \mathrm{~mm}$ gap between the edges.

Two types of stimulation sources are applied in the computational model. First, voltage stimulations are calculated to match the experimental settings. The voltages applied to the electrodes are normalized to $-1 \mathrm{~V}$ on the cathode and $0 \mathrm{~V}$ on the anode. Second, current stimulation sources on the electrodes are also studied. Conventionally, current stimulations are in ESCS computation. Thus, this allows us to make a direct comparison between ESCS and ISCS. In our computational model, current stimulations are $10 \mathrm{~mA}$ on the anode and $-10 \mathrm{~mA}$ on the cathode for ESCS, while the current amplitudes are $1 \mathrm{~mA}$ for ISCS.

The spinal cord is a complex and inhomogeneous system. In the volume conductor model for stimulation of it, four major domains are considered: the CSF, the white matter, the grey matter and the electrodes. In order to generate the distributions of the electrical current density in, and the electrical potential on the volume conductor, Laplaces Law $\left(\nabla^{2} \phi_{e}=0\right)$ and the generalized version of Ohms Law $(J=\sigma E, E=$ 


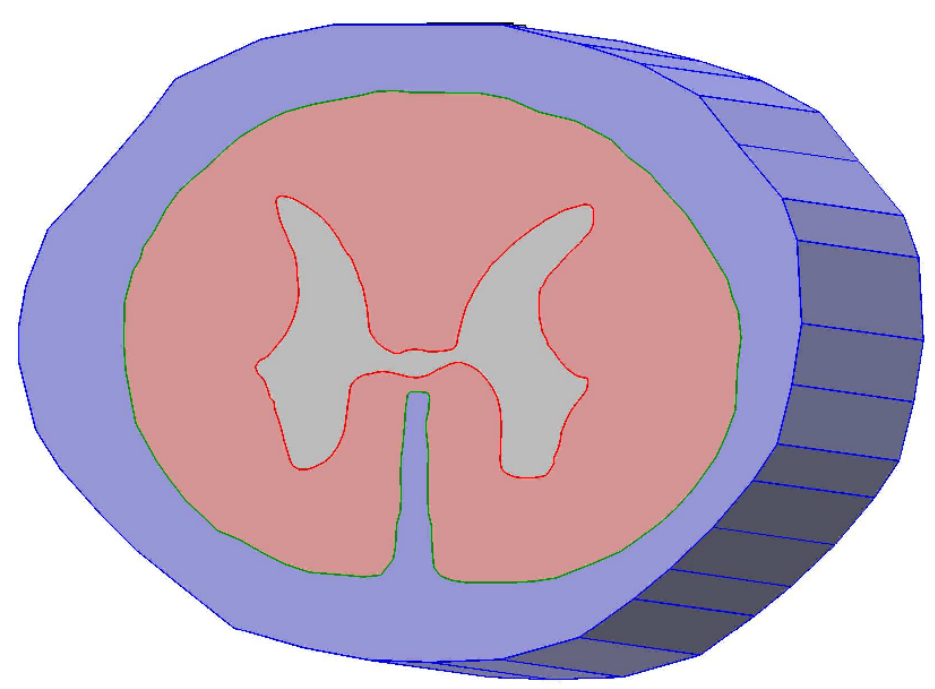

(a) anatomical structure

\section{$1 \mathrm{~mm}$}

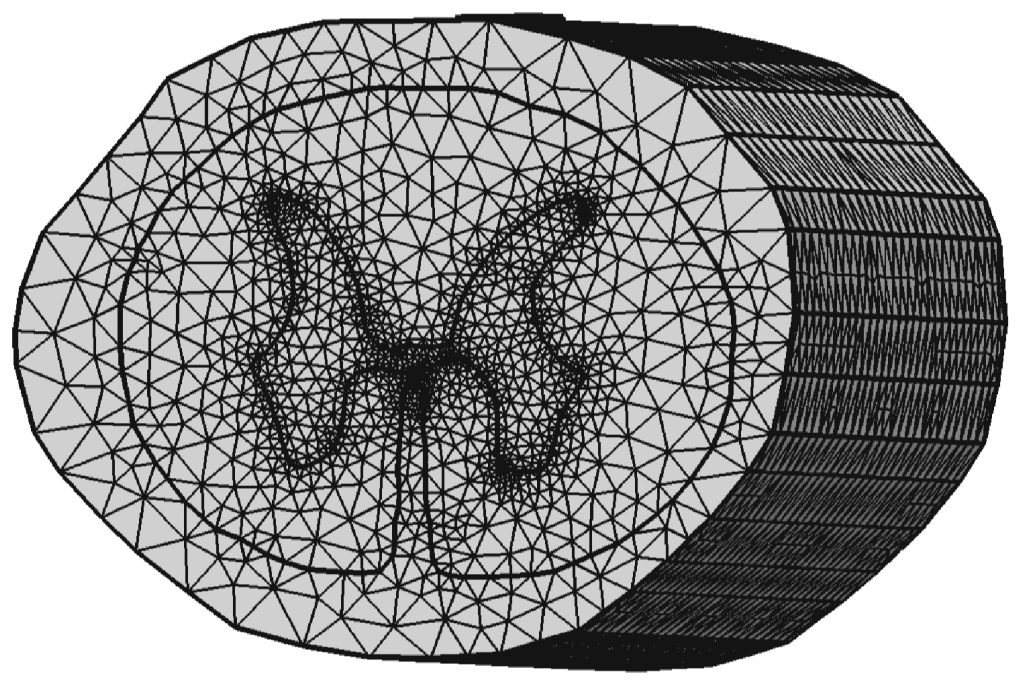

(b) mesh configuration

Figure 3.3: The three dimensional (a)anatomical structure (Blue: CSF; Red: White Matter; Grey: Grey Matter.) and (b)mesh configuration of spinal cord. The model has $20 \mathrm{~mm}$ length in vertical direction and about $10 \mathrm{~mm}$ transverse diameter. Physics controlled mesh setting is utilized here. Full description is in the literature [32]. 
$\left.-\nabla \phi_{e}\right)$ are used by the FEM algorithm as the governing equations, as described in detail below. Here $\phi_{e}$ is the electrical potential and the subscript e represents the external voltage outside the fiber nodes, $E$ is the electric field, $J$ is the current density, and $\sigma$ is the conductivity tensor reflecting the inhomogeneous resistivities within the spinal cord.

The electrical properties are characterized basically by the anisotropic electrical conductivity (Table 3.1) [33]. The conductivities of CSF and grey matter are reasonably isotropic and treated as such, but the conductivity of the white matter is very anisotropic, with higher axial conductivity. For simplicity, the electrodes are initially assumed to be made from platinum with a conductivity of $9.43 \times 10^{6} \mathrm{~S} / \mathrm{m}$ [34] (platinum electrodes will be used in the final-version clinical devices). The dura is surrounded by epidural fat and vertebral bone which have much lower electrical conductivities than CSF. Therefore, the outer surface of the dura is taken to be an insulator.

Comsol employs a physics controlled mesh, which adapts a suitable meshing method to match with the physical setting. In this case, a 3-D free tetrahedral mesh is used, as shown in Fig. 3.3. The element size depends on the local geometry in the range between $0.2 \mathrm{~mm}$ and $1.6 \mathrm{~mm}$, eg., boundaries and vertices with sharp angles will have a smaller mesh size. Because of the small size of the electrodes, a mesh that is finer than the normal default one is employed to achieve higher accuracy, although at the expense of computational efficiency. A more detailed description of the method is in the Comsol Manual [32].

The results of the computations for the voltage distribution on the spinal cord are exported as Cartesian coordinate matrix data for use in the nerve fiber model. The vertical step size is $0.5 \mathrm{~mm}$ and those along the cross-sectional directions are $0.1 \mathrm{~mm}$. 


\subsubsection{Nerve Fiber Model}

With the FEM predictions in hand, a model of the nerve fiber is then used to determine the internal depolarization voltage of each nerve node. We use the nerve fiber model of McNeal [18] which employs the electrical network shown in Fig. 3.4 to anatomically segment the nerve fiber. This method assumes that the radius of the nerve fiber is small enough so that the external voltage at any individual node is largely constant, and the presence of the nerve fiber does not influence the external electrical field.

The external electrical field calculated in the volume conductor model is essential for the nerve fiber model. The external voltages along the path of the nerve fiber are required to calculate the internal depolarization voltage. Fibers aligning vertically in the dorsal columns (DC) are represented by a parallel-cluster circuit model, wherein it is assumed that the individual fibers have uniform diameters of $5 \mu \mathrm{m}$. All the DC fibers are identical and are taken to be electrically independent, i.e., non-interfering

Table 3.1: Material Conductivity

\begin{tabular}{cc}
\hline Material & Conductivity $(\mathrm{S} / \mathrm{m})$ \\
\hline Cerebrospinal fluid & 1.7 \\
White Matter (longitudinal) & 0.6 \\
White Matter (transverse) & 0.08 \\
Grey Matter & 0.23 \\
Platinum Electrodes & $9.43 \times 10^{6}$ \\
\hline
\end{tabular}


with each other. For consistency across the calculations, a forty-node model is adopted in them, even though this is larger than the minimum number of nodes required, making the error caused by the finite length of the nerve fibers negligible. All other input parameters are based on the anatomical properties of the nerve fiber used by Coburn [25], as shown in Table 3.2. By applying conservation of current to the circuit shown in Fig. 3.4, we can derive an expression that describes how the rate of change of the depolarization voltage at node $j$ depends on the external voltage and on the depolarization voltages at the neighboring nodes. The starting point is equation (3.1) in which the terms on the left-hand side denote currents flowing out from node $j$ : the first term is the current from the capacitor discharge and the second term is ionic current going through the axon membrane. Meanwhile, the terms on the right-hand side of equation (3.1) represent currents flowing into node $j$ from neighbouring nodes. 

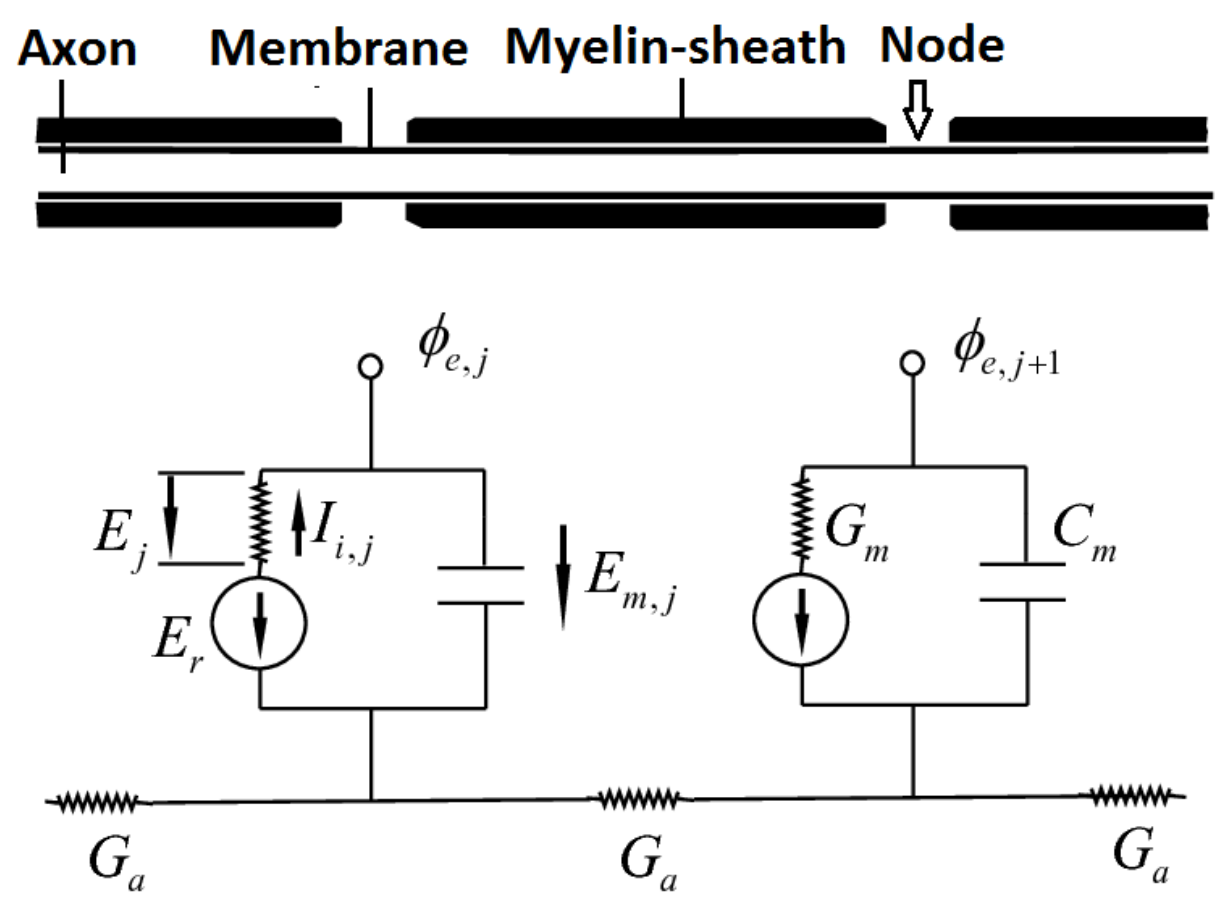

Figure 3.4: The circuit model used for fiber segmentation [25]. Subscripts $e$ and $j$ represent the external voltage at fiber node $j$. 


$$
C_{m} \frac{d E_{m, j}}{d t}+I_{i, j}=G_{a}\left(\phi_{i, j-1}-2 \times \phi_{i, j}+\phi_{i, j+1}\right)
$$

Under the subthreshold condition $G_{m}$ is widely accepted as a time independent constant $[25,18]$. After the threshold is reached, the membrane conductance $G_{m}$ begins to change with time, but the time scale for conductance change and the rate of nerve-action potential propagation is milliseconds $[16,17]$. In our study, the applied pulse duration is $20 \mu \mathrm{s}$, which is much shorter than the time scale mentioned there. Thus, 1) the membrane conductance can be safely accepted as a time independent constant even in the suprathrehold condition; 2) depolarization voltages on nodes in the pulse length are basically caused by the pulse stimulation rather than by potential propagation between nodes. The ionic current satisfies:

$$
I_{i, j}=G_{m} E_{j}
$$

From the circuit structure shown in Fig. 3.4, the voltage across the capacitor at node $j$ is the combination of the depolarization voltage $E_{j}$ and the nerve resting potential $E_{r}$. It is assumed that the resting potential is a time independent constant and the same for all nodes:

$$
E_{m, j}=E_{j}+E_{r}
$$

The desired expression can be written in terms of depolarization voltages and external voltages as

$$
\tau \dot{E}_{j}=E_{j-1}-(2+\gamma) E_{j}+E_{j+1}+\phi_{e, j-1}-2 \phi_{e, j}+\phi_{e, j+1}
$$

Here $\phi_{e, j}$ is the external voltage at node $j$, and 
Table 3.2: Physiological Parameters of Never Fibers

\begin{tabular}{cc}
\hline Parameter & Value \\
\hline$c_{m}$ membrane capacitance per centimeter square & $2 \mu \mathrm{Fcm}^{-2}$ \\
$g_{m}$ membrane conductance per centimeter square & $30.4 \mathrm{Scm}^{-2}$ \\
$l$ width of membrane at node of Ranvier & $2.5 \mu \mathrm{m}$ \\
$\rho_{i}$ axoplasm resistivity & $110 \Omega \mathrm{cm}$ \\
$D$ fiber diameter & $5 \mu \mathrm{m}$ \\
$d / D$ ratio of axon to fiber diameter & 0.7 \\
$L / D$ ratio of nodes distance to fiber diameter & 100 \\
\hline
\end{tabular}

$$
\begin{gathered}
\gamma=\frac{G_{m}}{G_{a}} \\
\tau=\frac{C_{m}}{G_{a}} \\
G_{a}=\frac{\pi d^{2}}{4 \rho_{i} L} \\
G_{m}=g_{m} \pi l d \\
C_{m}=c_{m} \pi l d
\end{gathered}
$$

The serial equations for the nerve fiber model with $\mathrm{n}$ nodes can be written in matrix form using the notation: 


$$
\tau \dot{E}=A E+F \phi_{e}
$$

Here, considering $n$ nodes on a nerve fiber: $E$ is a $n$ element column matrix of internal depolarization voltages; $A$ and $F$ are both $n \times n$ constant matrices based on the anatomical geometric and biophysical properties of the nerve fibers; $\phi_{e}$ is a $n$ element column matrix of the external voltages, which depends on the voltage waveform applied to the electrodes.

Equation (3.5) is a system of first order ordinary differential equations. The Laplace transform method is adopted to reach solutions.

Define:

$$
\begin{gathered}
E(t)=y(t), \\
M=\frac{A}{\tau}
\end{gathered}
$$

is a constant matrix, and

$$
Q=\frac{F}{\tau}
$$

is a constant matrix.

Then equation (3.5) can be rewritten as:

$$
\dot{y}(t)=M y(t)+Q \phi(t)
$$

Because the volume conductor model is stationary, the external voltage obtained from the volume conductor model has the same phase as the applied voltage on the electrodes. Thus, the external voltage can be represented by the product of the 
voltage amplitude and a rectangular wave with unit amplitude. The length of the rectangular wave is equals to the pulse length on the electrodes.

$$
\phi(t)=V G(t)
$$

Here, $V$ is the wave amplitude, which is the output of the electrostatic calculation from the volume conductor model. $G(t)$ is the rectangular wave, which is constructed of Heaviside step functions:

$$
G(t)=u(t-a)-u(t-b), a<b
$$

$u(t)$ is the Heaviside step function, $a$ and $b$ is the wave starting and ending time respectively.

Using the Laplace Transform to equation (3.6),

$$
L[\dot{y}(t)]=M \cdot L[y(t)]+Q V \cdot L[G(t)]
$$

We get:

$$
Y(s)=K(s) Q V G(s) .
$$

Here:

$$
\begin{aligned}
& G(s)=\frac{1}{s}\left(e^{-a s}-e^{-b s}\right) \\
& K(s)=(s \cdot I-M)^{-1} .
\end{aligned}
$$

Using the Inverse Laplace Transform

$$
y(t)=L^{-1}[G(s) K(s)] \cdot(Q V)
$$


Where

$$
\begin{aligned}
L^{-1}[G(s) K(s)] & =L^{-1}\left[\frac{K(s)}{s} e^{-a s}\right]-L^{-1}\left[\frac{K(s)}{s} e^{-b s}\right] \\
& =u(t-a) f(t-a)-u(t-a) f(t-a)
\end{aligned}
$$

Here:

$$
f(t)=L^{-1}\left[\frac{K(s)}{s}\right]
$$

and $K(s)$ and $f(t)$ are determined by Mathematica symbolic calculation.

Therefore,

$$
y(t)=[u(t-a) f(t-a)-u(t-b) f(t-b)] \cdot Q V
$$

The 3-D parallel-cluster nerve model covers all possible paths of the DC fibers. Axonal nodes have $500 \mu \mathrm{m}$ gap space according to above calculation. In reality, axonal nodes are staggered along the vertical direction. If the spinal cord is divided by into series of layers with $500 \mu \mathrm{m}$ gap, an axonal node can have a vertical offset to its nearest layer in the range from $0 \mu \mathrm{m}$ to $500 \mu \mathrm{m}$. Our study shows that this offset value has no significant impact on excitation results. Thus, it is simply assumed that the positions of the axonal nodes are not staggered: they are on the same planes with $500 \mu \mathrm{m}$ internode spacing. Color contour plots of the results found within each axial and sagittal cross-sectional slice are generated to show the depolarization voltages of the nodes at each level of the spinal cord in the next Chapter. A depolarization voltage of $25 \mathrm{mV}$ is taken as the threshold of successful stimulation [8]; therefore, a $25 \mathrm{mV}$ color contour is used to denote the limiting range of the excitation area. This approach allows us to investigate how the distance to the electrodes from the pial surface affects the stimulation results. 
Because the spinal cord is an extremely complicated neurological system with numerous nerve fibers and even more axonal nodes, the status of a single nerve fiber provides very little information about the overall functionality of SCS within a given situation. Therefore, it is useful to introduce a statistical approach that can help to sharpen the analysis process. Combined with the three-dimensional depolarizationvoltage model described above, this method can provide the depolarization voltage distribution on the nodes, the number of nodes of different depolarization strength, and the geometric distribution of the excited nodes. This allows for a more comprehensive understanding of the SCS process within the spinal cord segment under study.

As shown in Chapter Four, from the excitation threshold of $25 \mathrm{mV}$ to the maximum depolarization voltage, contours curves are separated in $5 \mathrm{mV}$ steps. In the real case the saturation depolarization voltage is about $100 \mathrm{mV}$, thus nodes with depolarization voltage higher than the saturation voltage will be assigned the depolarization voltage of $100 \mathrm{mV}$ [35].

For ISCS, the stimulator electrodes are in direct contact with the white matter whereas for the ESCS they are on the dura and, as expected, this difference significantly changes the predictions of the volume conductor model. The color contour cross sections provide insight into the distribution of depolarization voltages on the surface of and within the spinal cord rather than on only a single nerve fiber. By combining these approaches, we are able to investigate excitation processes within specific SC zones and compare the results with those found in experimental tests of SCS on large animal models, as described below. The goal, in part, is to render the results in such a way that the contour plots provide intuitive visual information about the intensity and degree of focus of the stimulation, and thus help inform the design of the implant itself and the protocol for use. 


\section{Chapter 4}

\section{Results and Discussion}

In this chapter, modeling results from both the current stimulation and the voltage stimulation approaches are discussed in detail. Results of current stimulation are the natural extension of conventional ESCS modeling which always uses current stimulation. Besides comparing ESCS VS. ISCS stimulation results, a theoretical model is proposed to explain the experimental results obtained for the case of voltage stimulation.

\subsection{Current Source Stimulation}

As mentioned in Chapter Three, current source stimulations are applied to study the response of nerve fibers. For ESCS, current amplitudes are $10 \mathrm{~mA}$ on the anode and $-10 \mathrm{~mA}$ on the cathode. While for ISCS, without the blocking of CSF, 1 mA current amplitude already generates comparable but larger neural response than ESCS. Thus, $10 \mathrm{~mA}$ and $1 \mathrm{~mA}$ current amplitudes, rather than equal amplitudes, are applied for ESCS and ISCS respectively. 


\subsubsection{Electrical Potential Field in the Spinal Cord}

The external potential field and fiber-node depolarization voltage distribution has been modeled in the spinal cord. First of all, as the result of stimulation current, the voltage potential field on the spinal cord is determined through FEM (Fig. 4.1). For ISCS, the potential field has a larger maximum intensity beyond $0.25 \mathrm{~V}$ and a higher focused gradient at the areas close to electrodes. In contrast, because of the shunting effect caused by CSF in ESCS, the potential is more uniformly distributed in the range between $-1.5 \mathrm{~V}$ to $1.5 \mathrm{~V}$ and the potential field gradient is much smaller than that of ISCS. Ratty's work shows that, from the point of view of external factors of fibers, a large vaule of the second order spatial derivative of the potential field is critical for fiber excitation $[20,21]$. At the aspect of extracellular potential field distributions, this result implies that ISCS exceeds ESCS at stimulation effectiveness and accuracy. This prediction is validated by the computational results for the membrane depolarization voltages which will be discussed in the next subsection. 


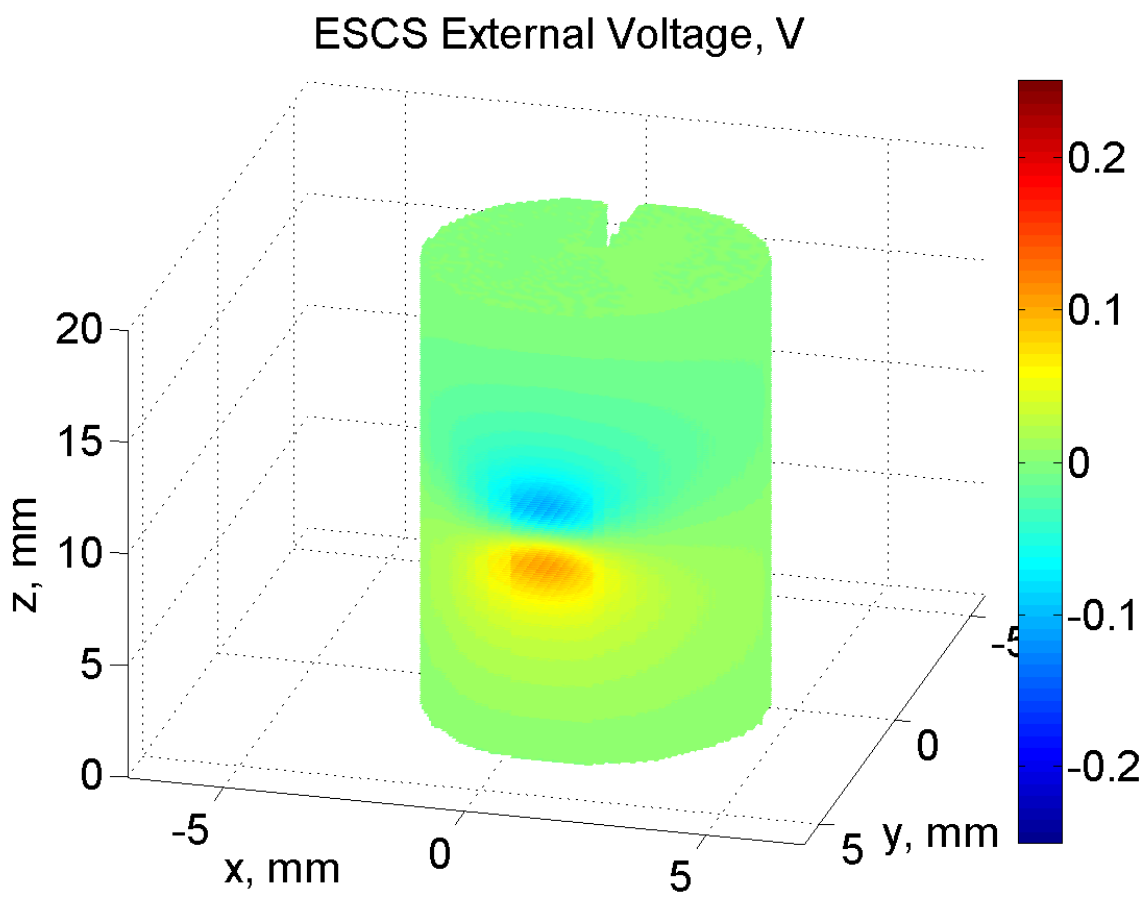

(a) ESCS $10 \mathrm{~mA}$

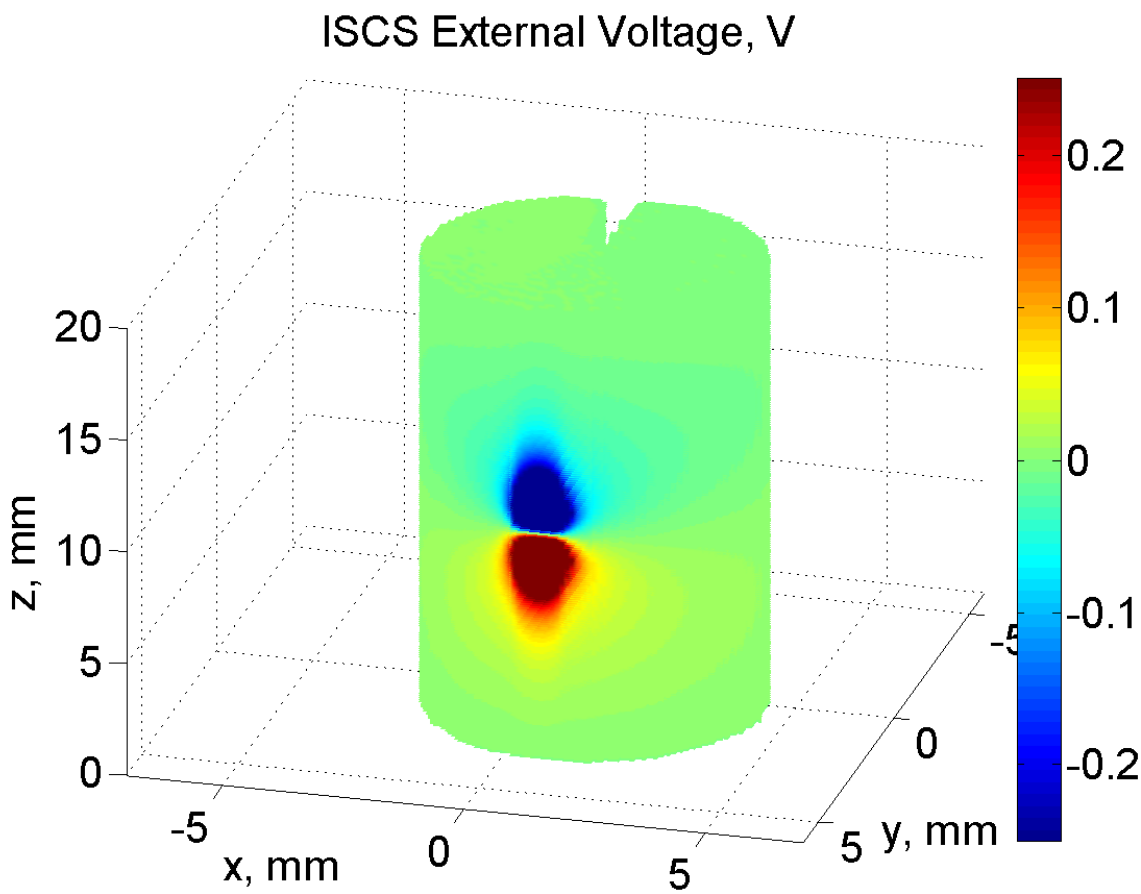

(b) ISCS $1 \mathrm{~mA}$

Figure 4.1: The potential field on the surface of the spinal cord for both case of the (a) ESCS with $10 \mathrm{~mA}$ stimulations and the (b) ISCS with $1 \mathrm{~mA}$ stumulation; the color represents the amplitude of the depolarization voltage. 


\subsubsection{Membrane Depolarization Voltage Distribution}

The obtained voltage distributions were employed as the inputs in the nerve fiber model. In this model, nerve fibers are converted into electrical circuits having resistances and capacitances which depend on the anatomical and physiological properties of the nerve fiber. The depolarization voltage is then calculated to determine whether or not the nerve fiber is excited.

Rather than looking at the whole spinal cord in this model, we focus only on an area on spinal cord with excited depolarization voltages. The highest depolarization voltage happens at the cross section on the same level as the cathode. The color contour plots represents depolarization distribution on the cross section. The contour starts from the boundary of $0 \mathrm{mV}$ depolarizatoin voltage and contour curve gradient increases by $5 \mathrm{mV}$ at each step, to saturate of $100 \mathrm{mV}$ [35]. Fig. 4.2 shows the ISCS depolarization voltages on $X-Y$ cross sections with different vertical values. The maximum excitation intensity and the largest excitation area are located on the cross section at the same vertical level of the cathode. At the cathode position, each depolarization contour includes a slightly larger area than the neighbouring layers for ISCS. The results for ESCS show the same property of reaching maximum excitation at the cathode level, and the contour area is more sensitive to a change in vertical distance from the cathode because of the shunting effect of the CSF (Fig. 4.3). For the case of ESCS, $10 \mathrm{~mA}$ current stimulation was applied to the electrodes, but only a slight area close to the stimulator is excited. However, even when a 10 times smaller current amplitude was applied to ISCS, there is a significantly larger response from the nerves in terms of number of fibers involved and depolarization intensity. Note that a considerable area reaches saturation depolarization. Thus, there is still room left to further decrease the stimulation current amplitude for the case of ISCS. This is more clearly illustrated in Fig. 4.4. Fig.4.4 shows the depolarization voltage profile 
of a single nerve fiber in the target area ( the nerve fiber is perpendicular to the cross section in Fig. 4.3 and intersects at the point at $x=0 \mathrm{~mm}$ and $y=0.27 \mathrm{~mm}$ ). The 100 $\mathrm{mV}$ saturation depolarization voltage limitation is not applied in the voltage profile. It shows that for both ESCS and ISCS the maximum depolarization is at the 11th node which is on the same level as the cathode. In contrast, the 7th node on the same level as the anode has the maximum hyperpolarization voltage. The excitation status depends on the maximum depolarization voltage among axon nodes. According to Fig 4.4, the maximum depolarization of ESCS is less than $40 \mathrm{mV}$ with $10 \mathrm{~mA}$ stimulation. But with only $1 \mathrm{~mA}$ stimulation, the ISCS has maximum depolarization more than $600 \mathrm{mV}$. In order to demonstrate the stimulation effectiveness, the depolarizations in Fig. 4.4 are not truncated by the $100 \mathrm{mV}$ saturation depolarization voltage. 


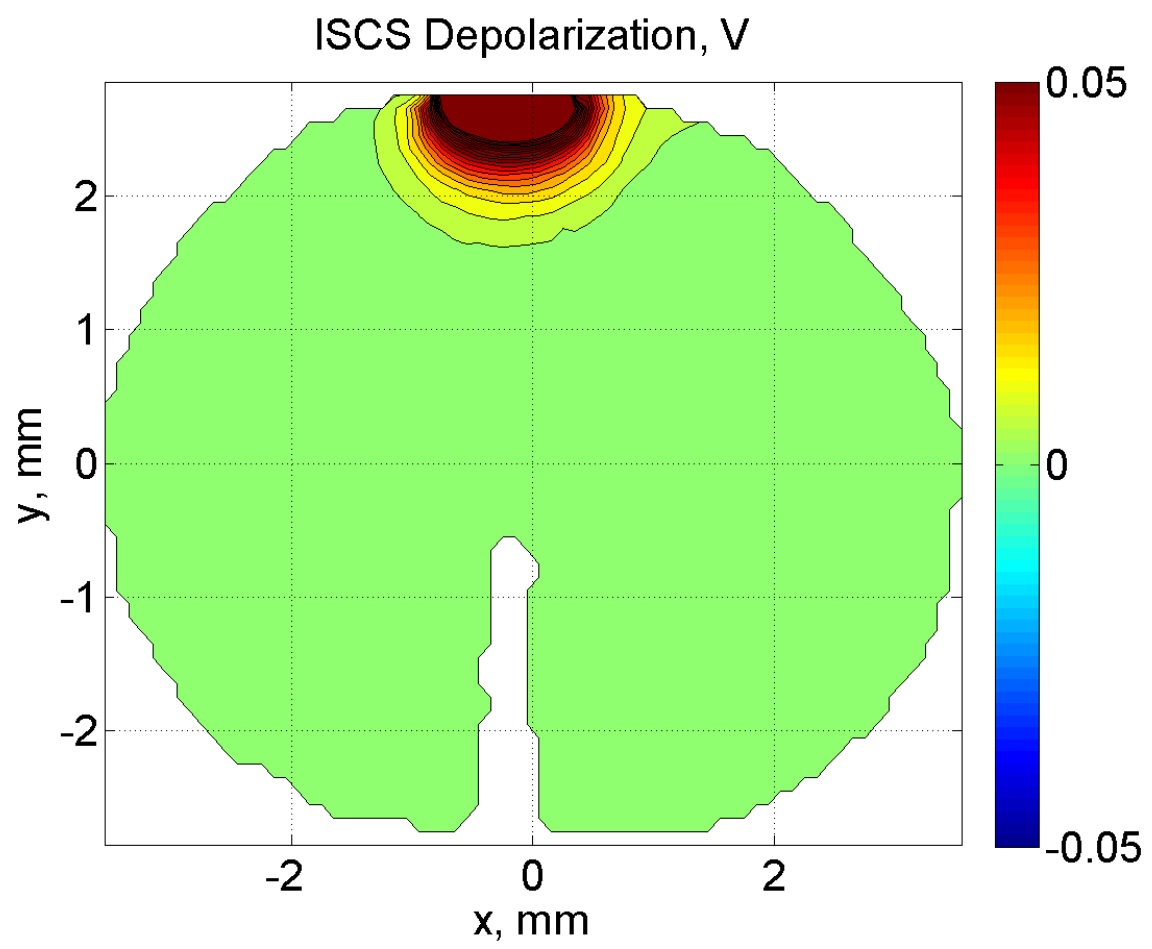

(a) ESCS

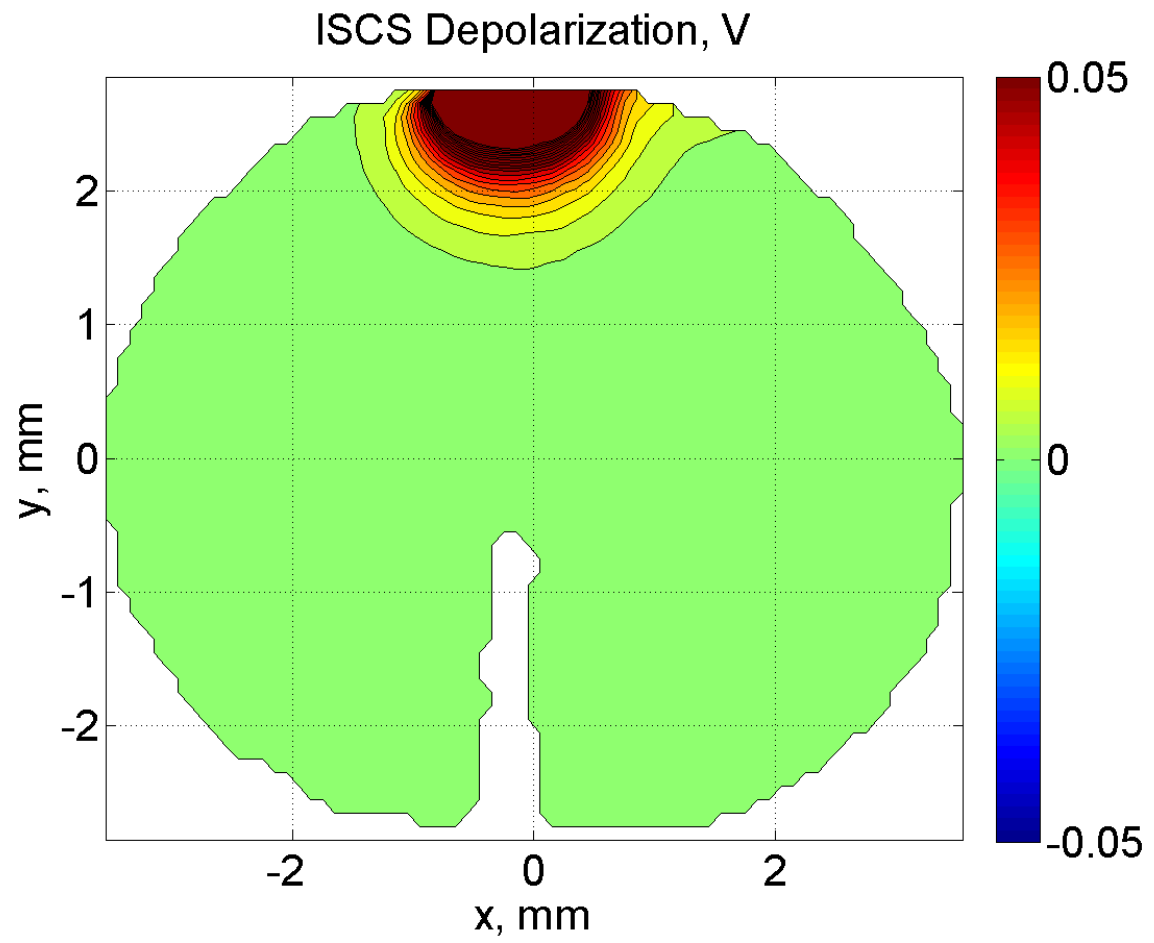

(b) ISCS

Figure 4.2: Continuing 


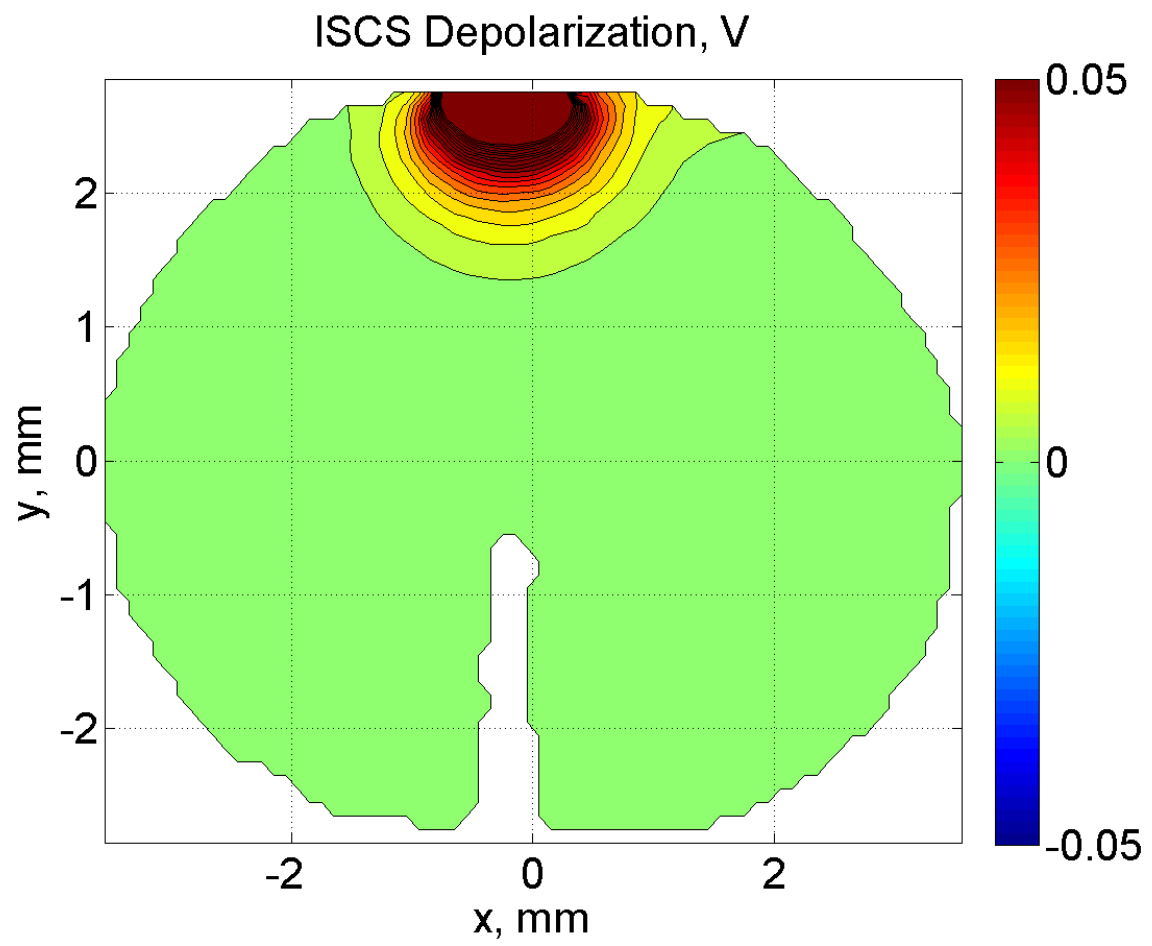

(c) ISCS

Figure 4.2: The depolarization voltage on cross sections of (a) $0.5 \mathrm{~mm}$ above the cathode (b) on the same level as the cathode (c) $0.5 \mathrm{~mm}$ below the cathode; the color represents the amplitude of voltage in units of volts. 


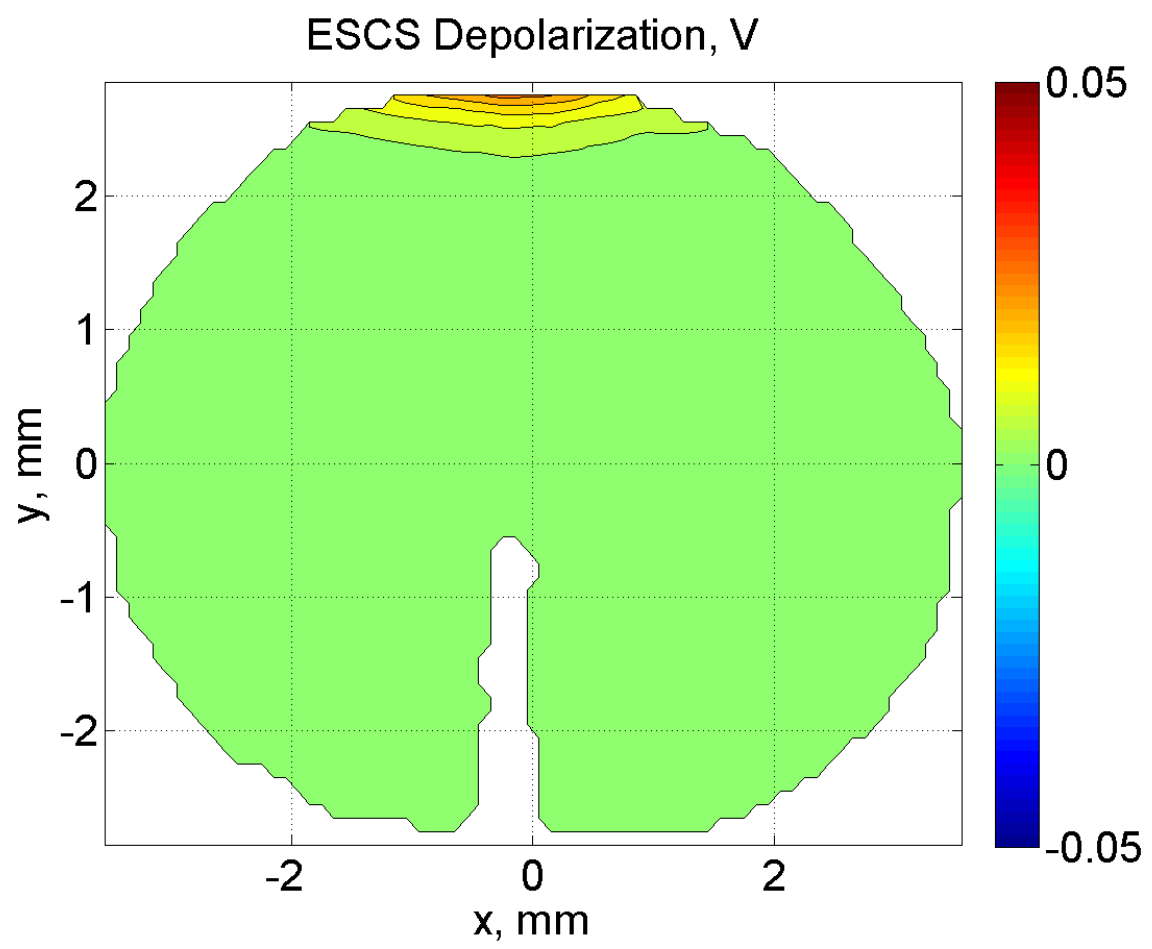

(a) ESCS

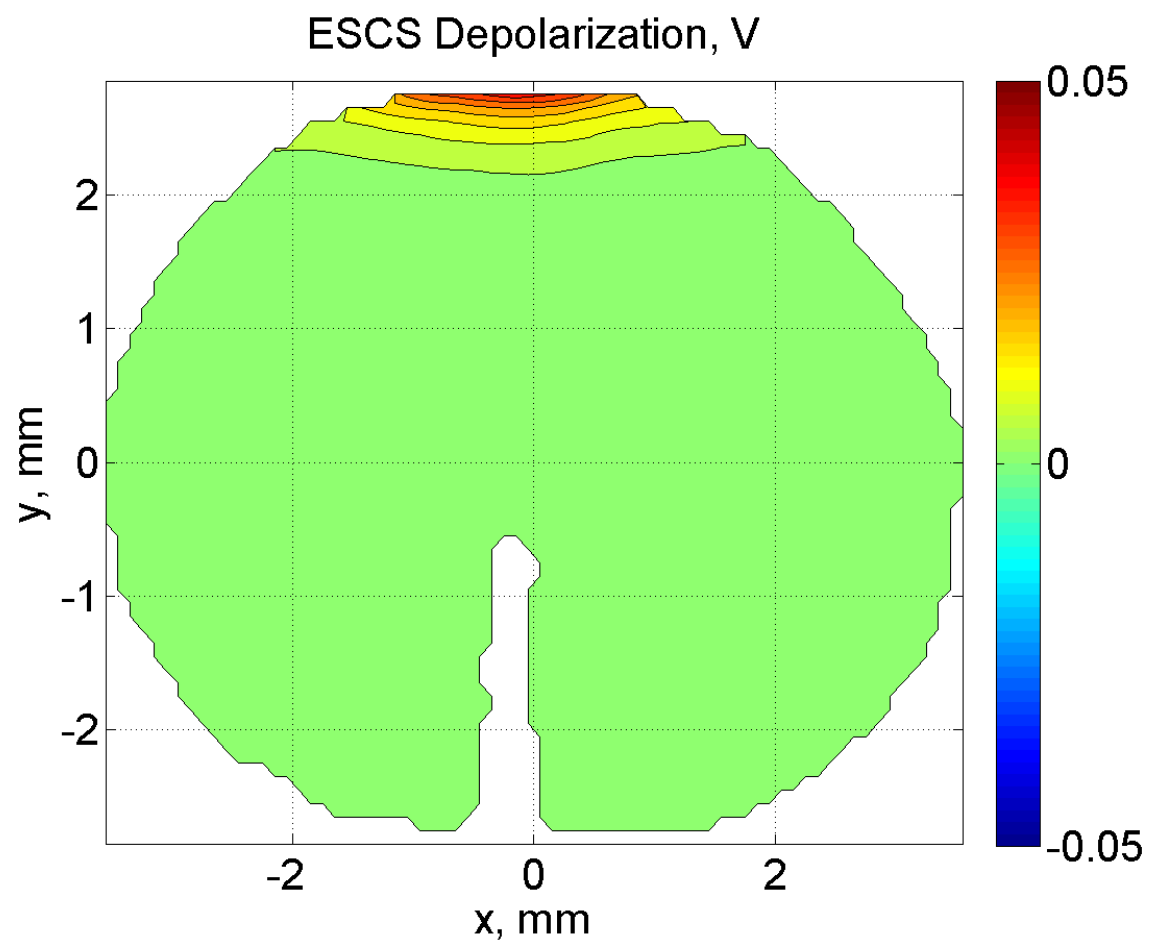

(b) ISCS

Figure 4.3: Continuing 


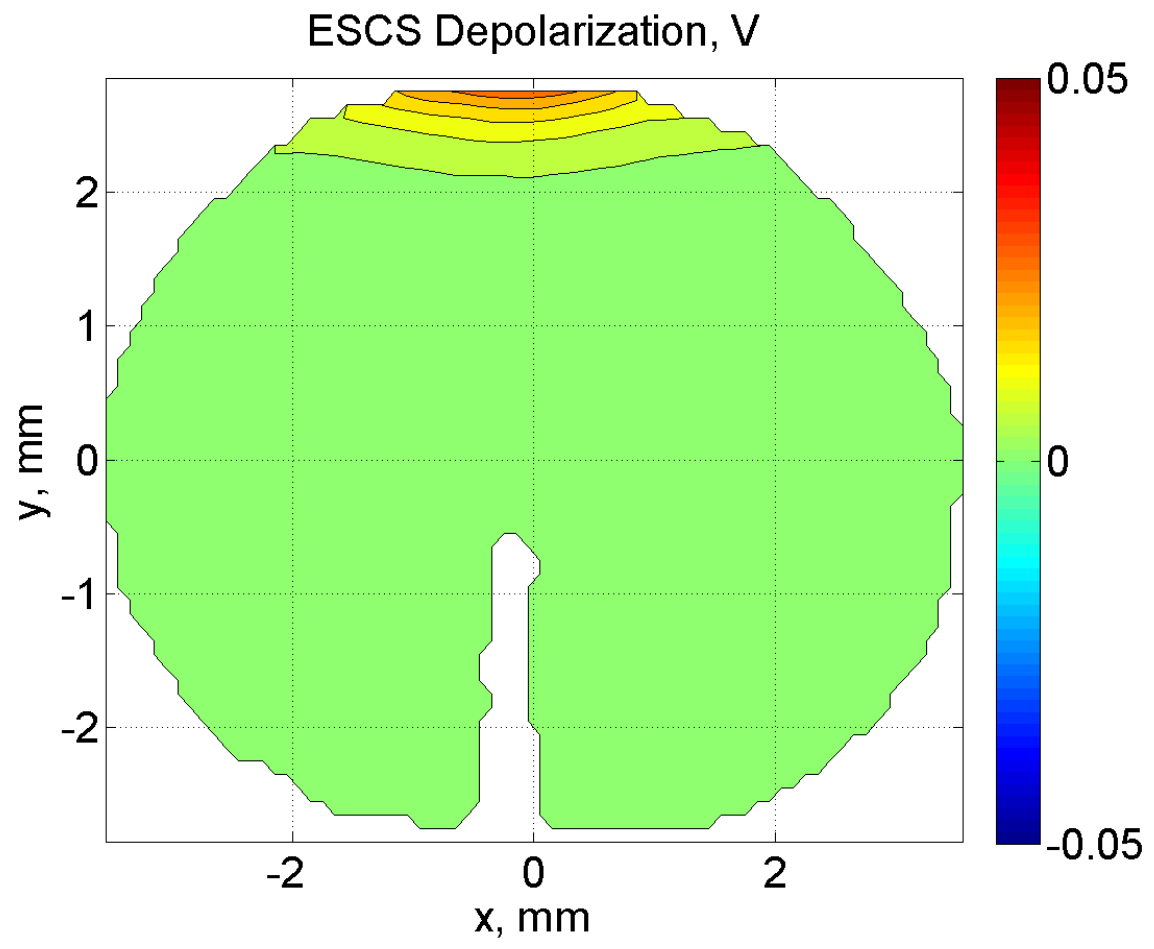

(c) ISCS

Figure 4.3: The depolarization voltage on cross sections at (a) $0.5 \mathrm{~mm}$ above the cathode (b) on the same level as the cathode (c) $0.5 \mathrm{~mm}$ below the cathode; the color represents the amplitude of voltage in units of volts. 


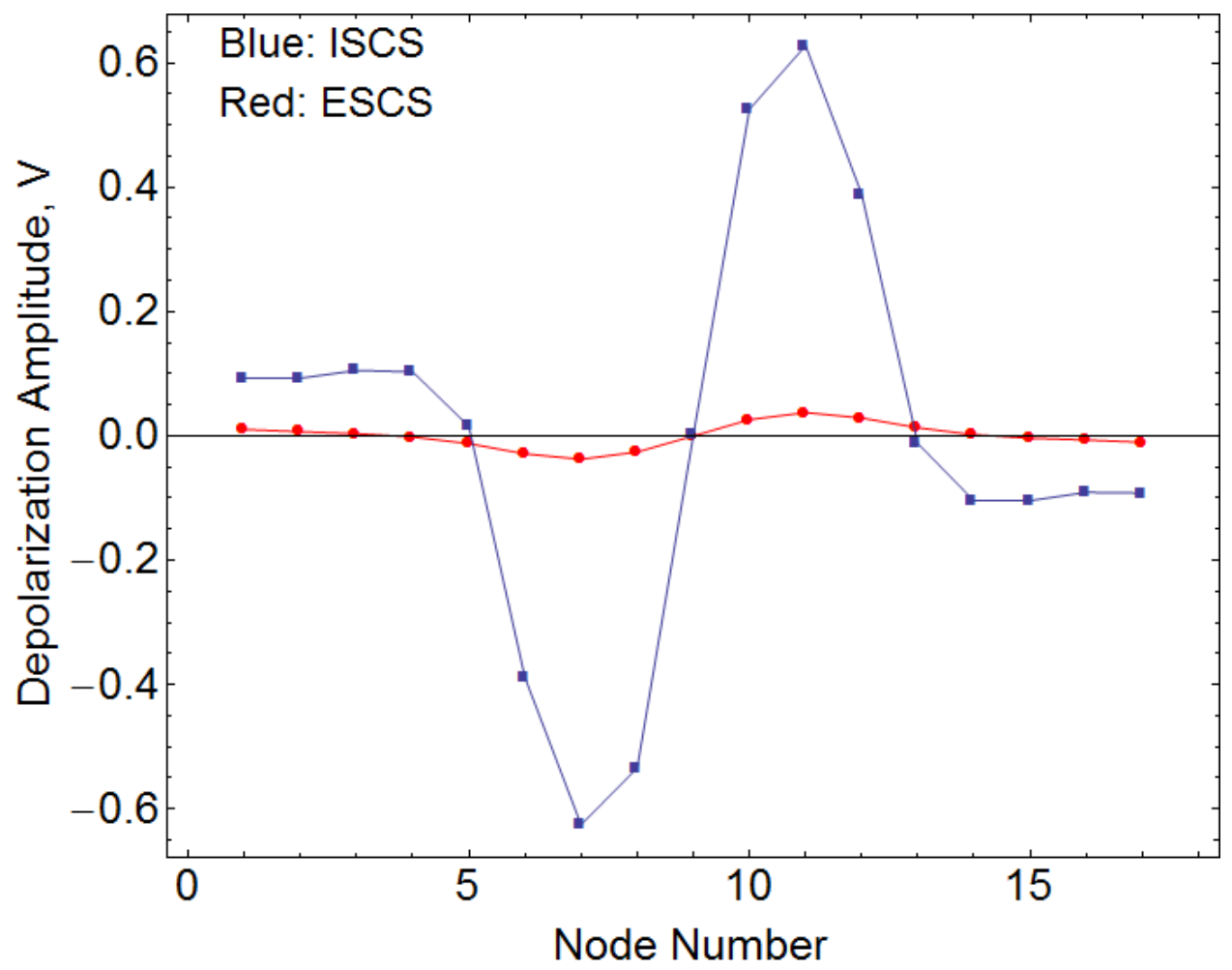

Figure 4.4: The depolarization on each axonal node of the target nerve fiber for the case of ESCS and ISCS. Red: ESCS; Blue: ISCS. 
By comparing the maximum depolarization in horizontal cross sections of ISCS and ESCS, it becomes clear that ISCS results in a large excitation area near the cathode. The excitation area has the shape of semi-circle in which about $50 \%$ of the area reaches saturation depolarization. In contrast, even with 10 times larger current amplitude, the excitation area is a narrow and long strip crossing several several sensory zones with only a modest depolarization voltage, which is less than $50 \mathrm{mV}$. A higher maximun depolazation provides a wider therapeutic window for stimulation. In other words, a lower stimulation voltage is required by ISCS than ESCS. Moreover, the excitation area of ESCS spreads along the $X$ direction coving almost the whole lateral section of the spinal cord. The lateral sections at the direction of one o'clock and eleven o'clock are the dorsal root entry zones, which should be prevented from excitation to void side effects. In ISCS, the excitation area is similar to a semi-circle. Extensive modeling results shows that the radius of the circle can be reduced by decreasing the stimulation voltage, which leads to a focused intensive excitation spot on the targeted nerves. This property indicates that ISCS has significant advantages in diminishing treatment side effects. However, the dilemma of ESCS is that the targeted nerves can not be successfully stimulated if the stimulation current is decreased to void unwanted stimulation. 


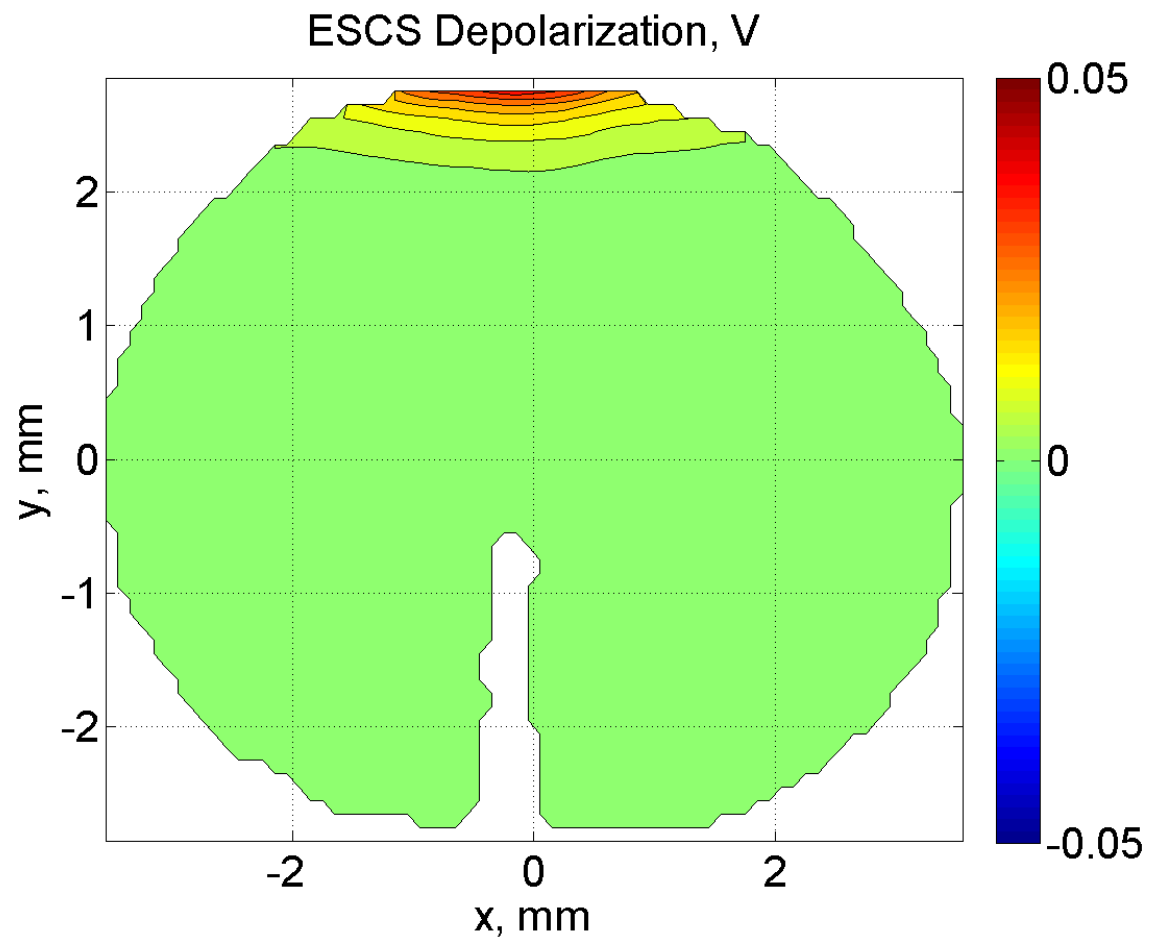

(a) ESCS $10 \mathrm{~mA}$

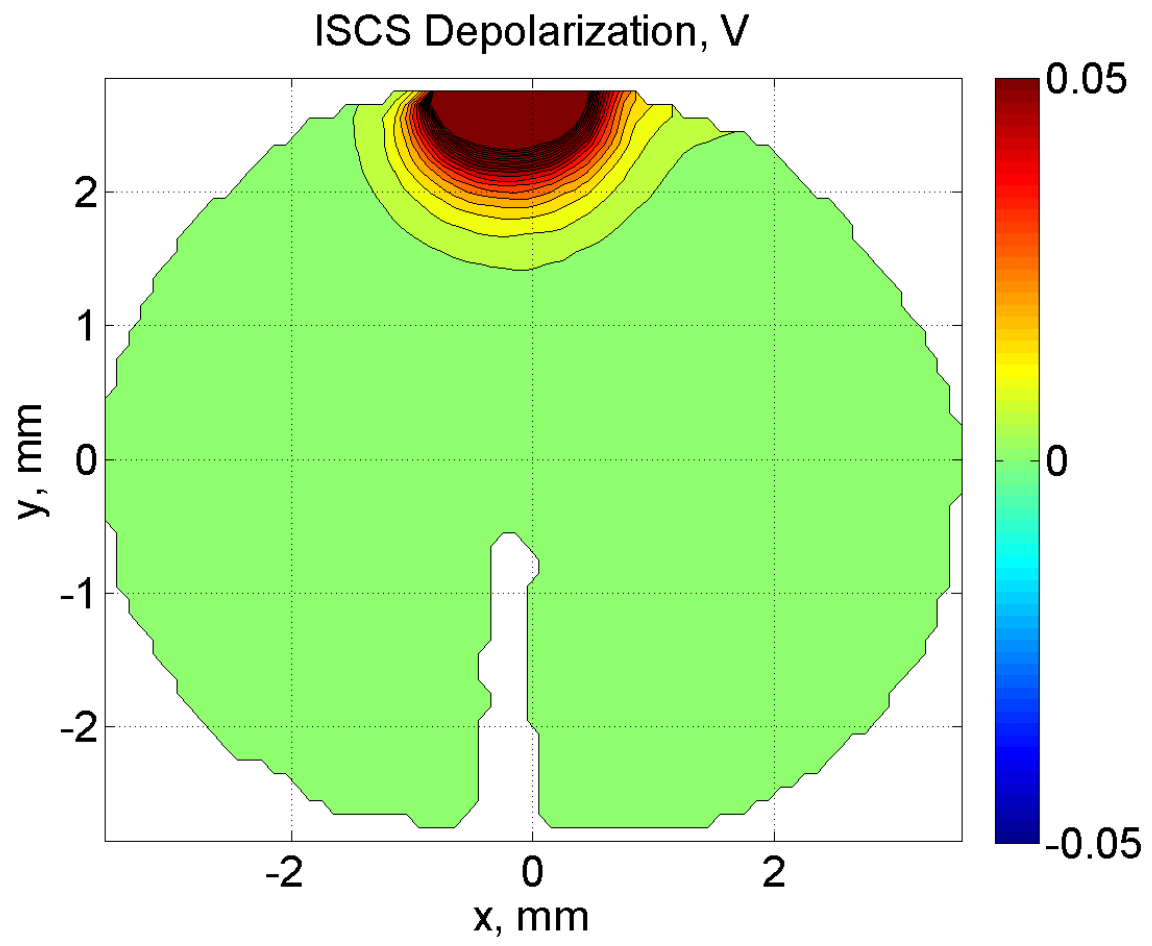

(b) ISCS $1 \mathrm{~mA}$

Figure 4.5: The depolarization voltage within cross sections on the same level as the cathode: (a) ESCS with $10 \mathrm{~mA}$ stimulation and (b) ISCS with $1 \mathrm{~mA}$ stimulation; the color represents the amplitude of depolarization voltage. 
For cross sections parallel to the $Y-Z$ plane, in ESCS, depolarization and hyperpolarization area originate at the electrodes postions and spread to levels beyond the electrodes (Fig. 4.6). In the case of ISCS, the depolarization area is filled by high intensity excitation, i.e., above $50 \mathrm{mV}$ as shown on the color legend. From the anatomical aspect, the $Y-Z$ plane goes through the central part of doral column, which is the excitation target. Therefore, a high intensity excitation is more desirable. Notice that levels right above the cathod are considerably hyperpolarized, even in the opposite direction of the anode on the $Z$ axis. The reason is that, in the case of ISCS, the voltage gradient on the spinal cord is greater than that of ESCS because of the direct contact between the electrodes and the spinal cord. This hyperpolarization effect can be applied to improve the excitation accuracy along the $Z$ direction, because different levels of the spinal cord connect to nerves reaching different parts of the body. 


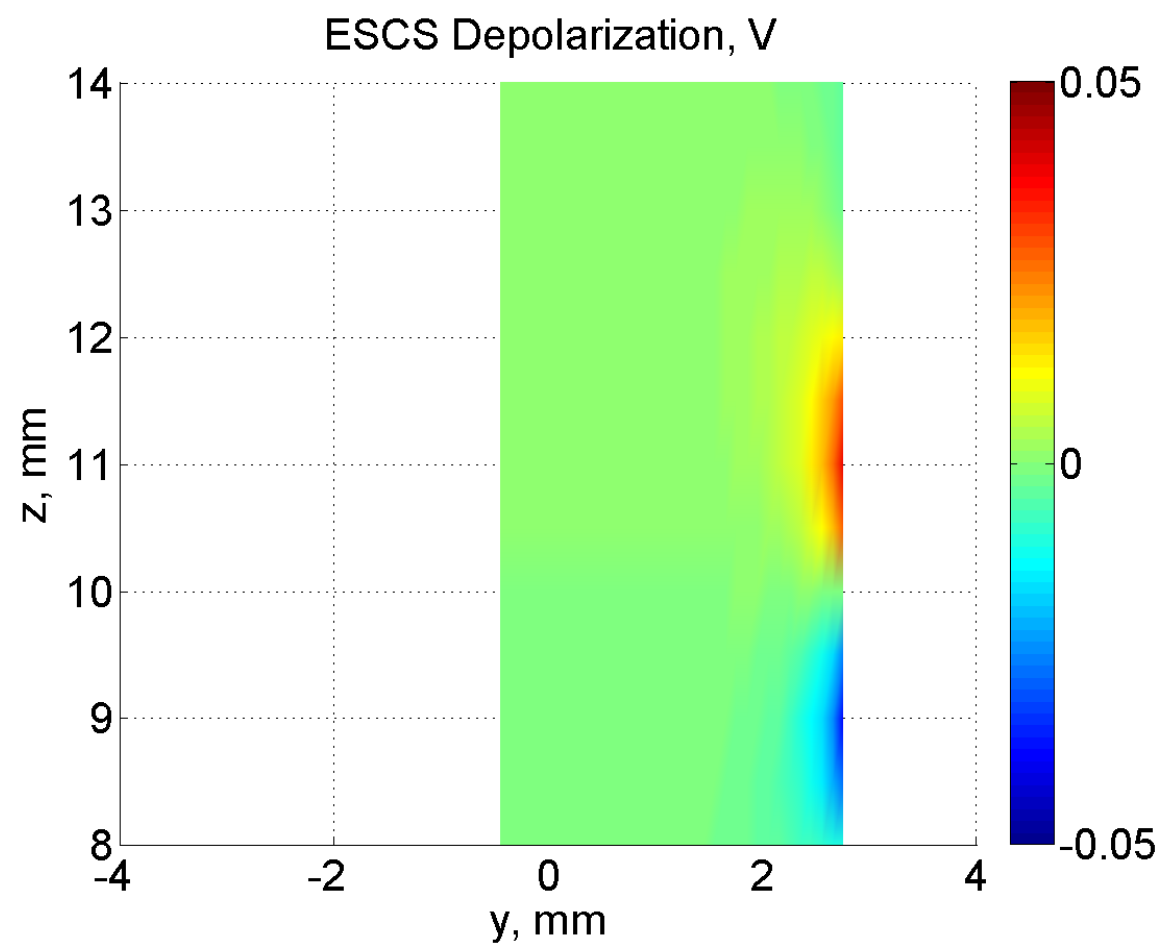

(a) ESCS

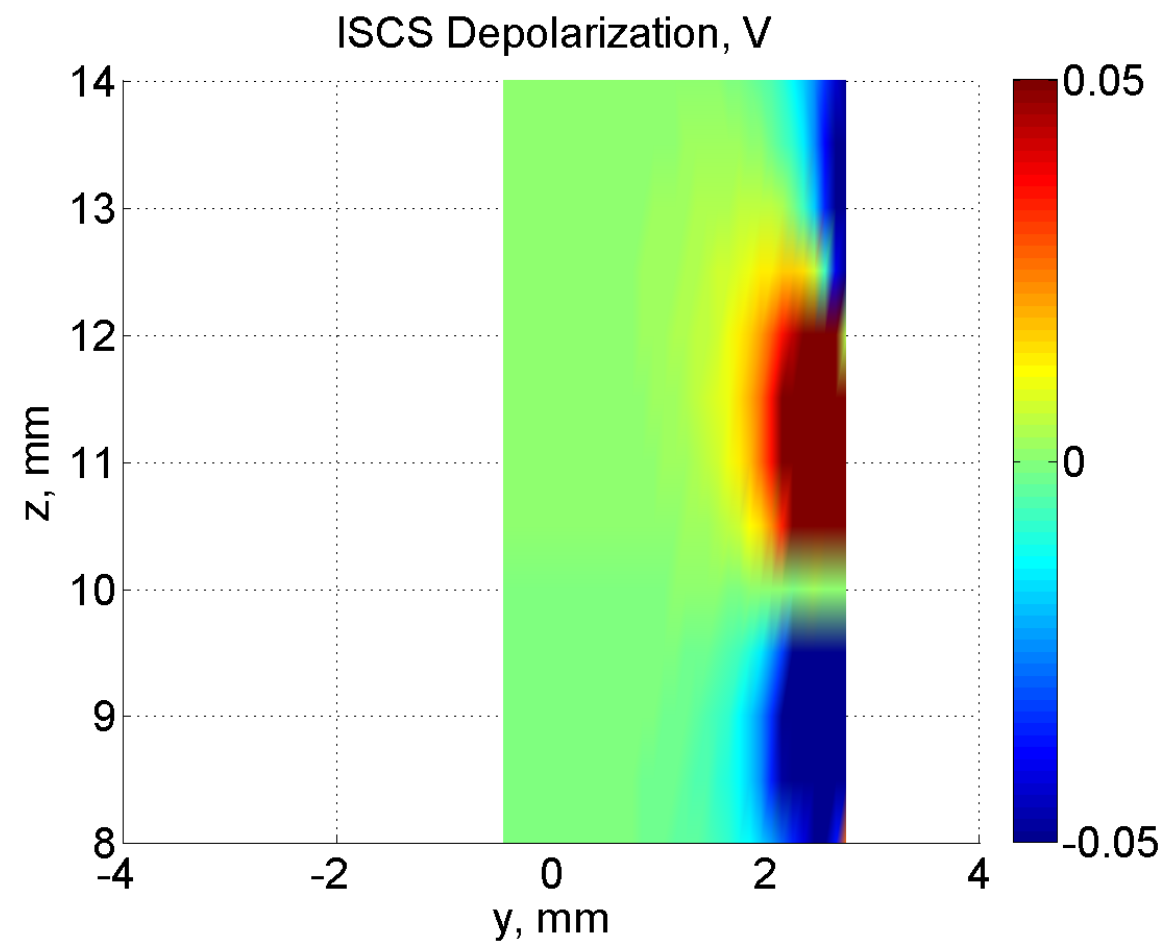

(b) ISCS

Figure 4.6: The depolarization voltage of (a) ESCS and (b) ISCS; the color represents the amplitude of voltage. Cross sections parallel to the $Y-Z$ plane and through the center of the spinal cord. 


\subsection{Voltage Source Stimulation}

In this section, similar to current source stimulation, the electrical potential field on spinal cord and the membrane depolarization voltage distribution are demonstrated. Additionally, computational results are compared with experimental results from the studies of the University of Iowa. A theoretical model is proposed to explain the experiment results.

\subsubsection{Electrical Potential Field in the Spinal Cord Generated by Voltage Source Stimulation}

For the voltage source stimulation, the anode is at ground and the voltage amplitude on the cathode is normalized to $1 \mathrm{~V}$. This is different from the case of current source stimulation in which the ground potential is at the middle layer between the anode and the cathode. According to Ratty's theory and our extension of it via computational results, the second order spatial derivative of the electrical potential field determines the depolarization voltages on the neural nodes. Thus, the ground potential reference is not critical.

Similar to current source stimulation, the voltage potential on the spinal cord is calculated by FEM (Fig. 4.7). Because electrodes have fixed potentials, in the case of ISCS, the potential field has extremely an large gradient focused on areas in contact with the cathode, and most of the area on spinal cord is not influenced because the potential is near $0 \mathrm{~V}$. In contrast, because of the shunting effect caused by CSF in ESCS, the whole spinal cord is effected: the potential amplitudes shift way from $0 \mathrm{~V}$ to the $0.3-0.5 \mathrm{~V}$ range and the potential field gradient is much smaller than that of ISCS. 


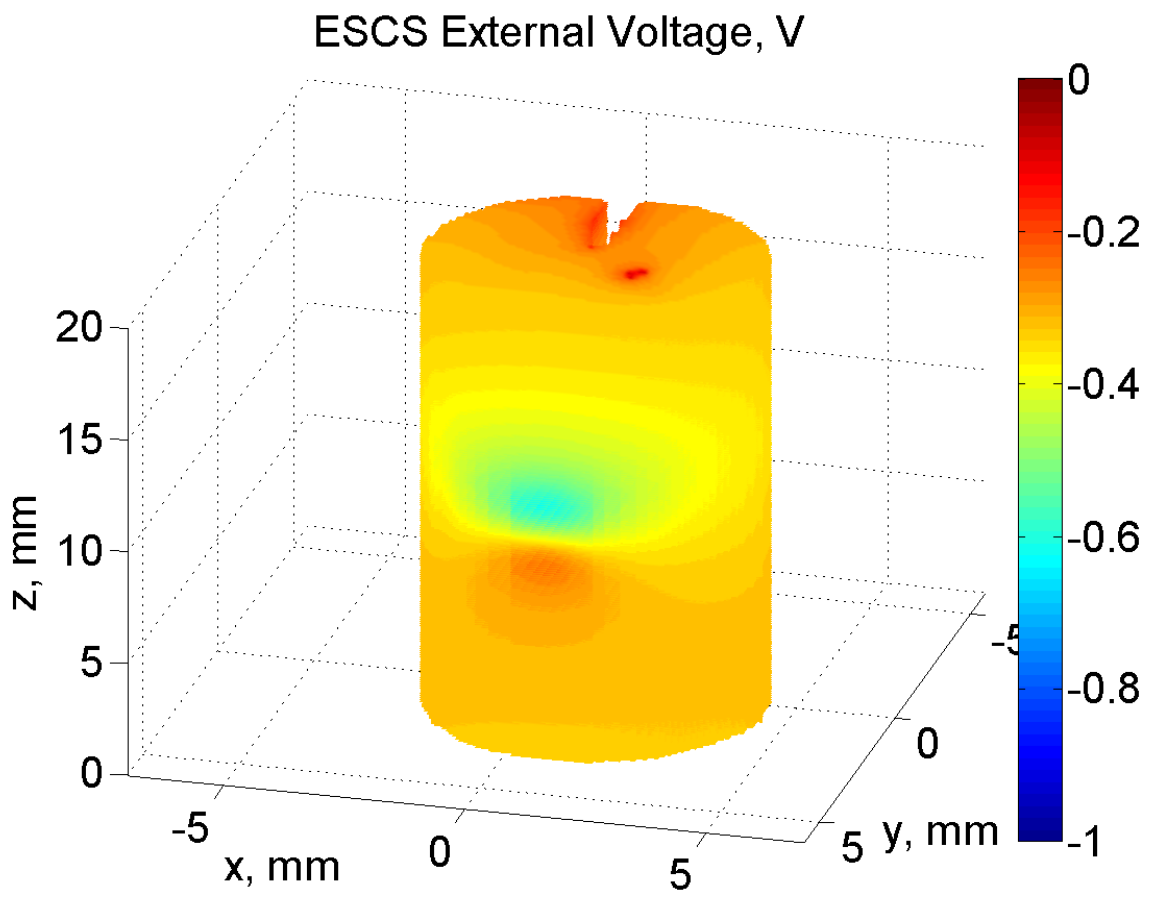

(a) ESCS

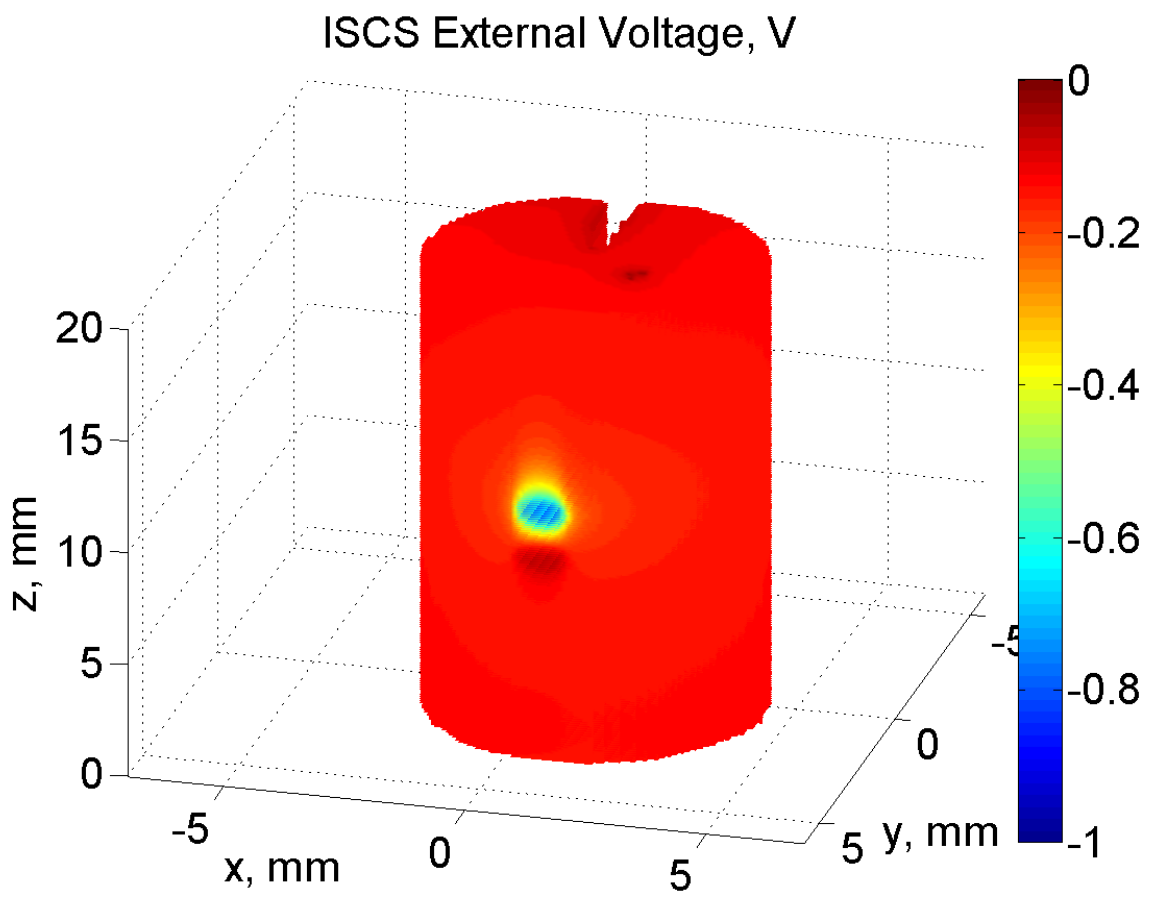

(b) ISCS

Figure 4.7: The potential field on the surface of spinal cord for both case of (a) ESCS and (b) ISCS; the color represents the amplitude of voltage in the units of volts. 


\subsubsection{Membrane Depolarization Voltage Distribution}

The depolarization voltages presented in the following graphs (Fig. 4.8, 4.9, 4.10, 4.11) are illustrated in a manner identical to the current source stimulation in terms of the color contour scales, positions of the cross sections and, plot sequence. Fig. 4.8 and Fig. 4.9 show that the $X-Y$ cross section with maximum excitation intensity and largest excitation area goes through the cathode for both ISCS and ESCS. The layers with maximum stimulation are compared in Fig. 4.10. Fig. 4.11 shows the $Y-Z$ cross section going through the center of the spinal cord.

These results demonstrate that in the case of ISCS, the depolarization voltage distribution for voltage source stimulation is identical to that of current source stimulation in terms of excitation area, intensity, and contour shape. For ESCS, voltage source stimulation and current source stimulation have similar excitation area and contour shape. The voltage source stimulation has slightly higher depolarization intensity than current source stimulation, but much smaller than for any instance of ISCS. 


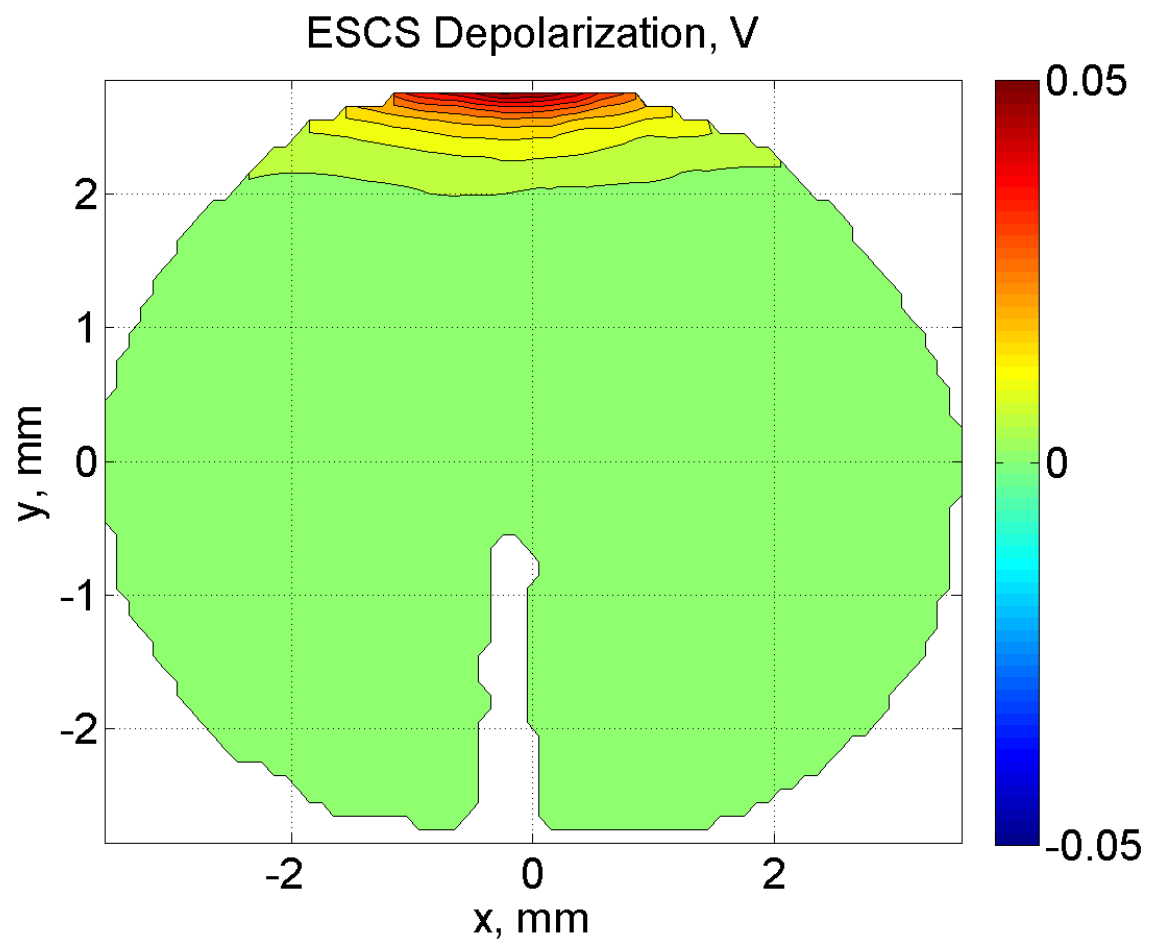

(a) ESCS

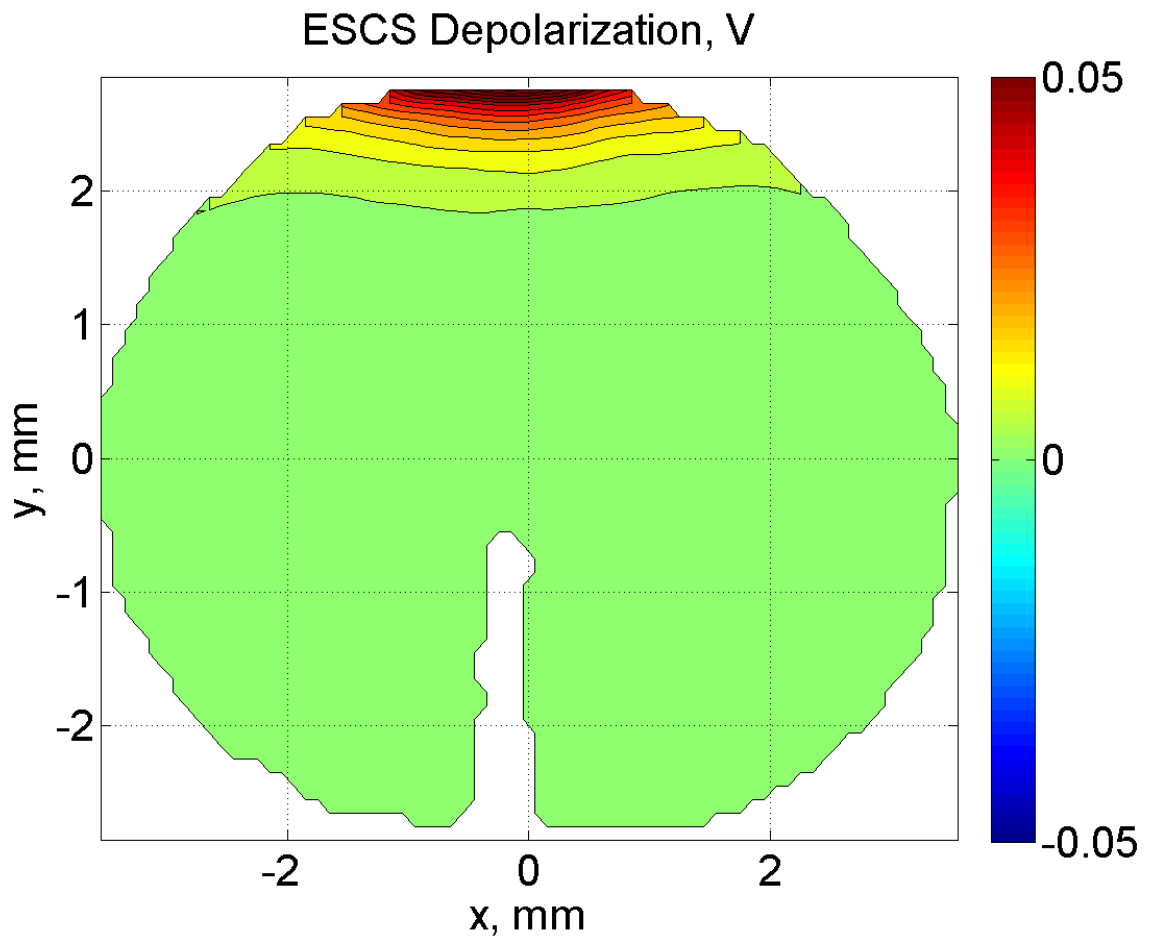

(b) ISCS

Figure 4.8: Continuing 


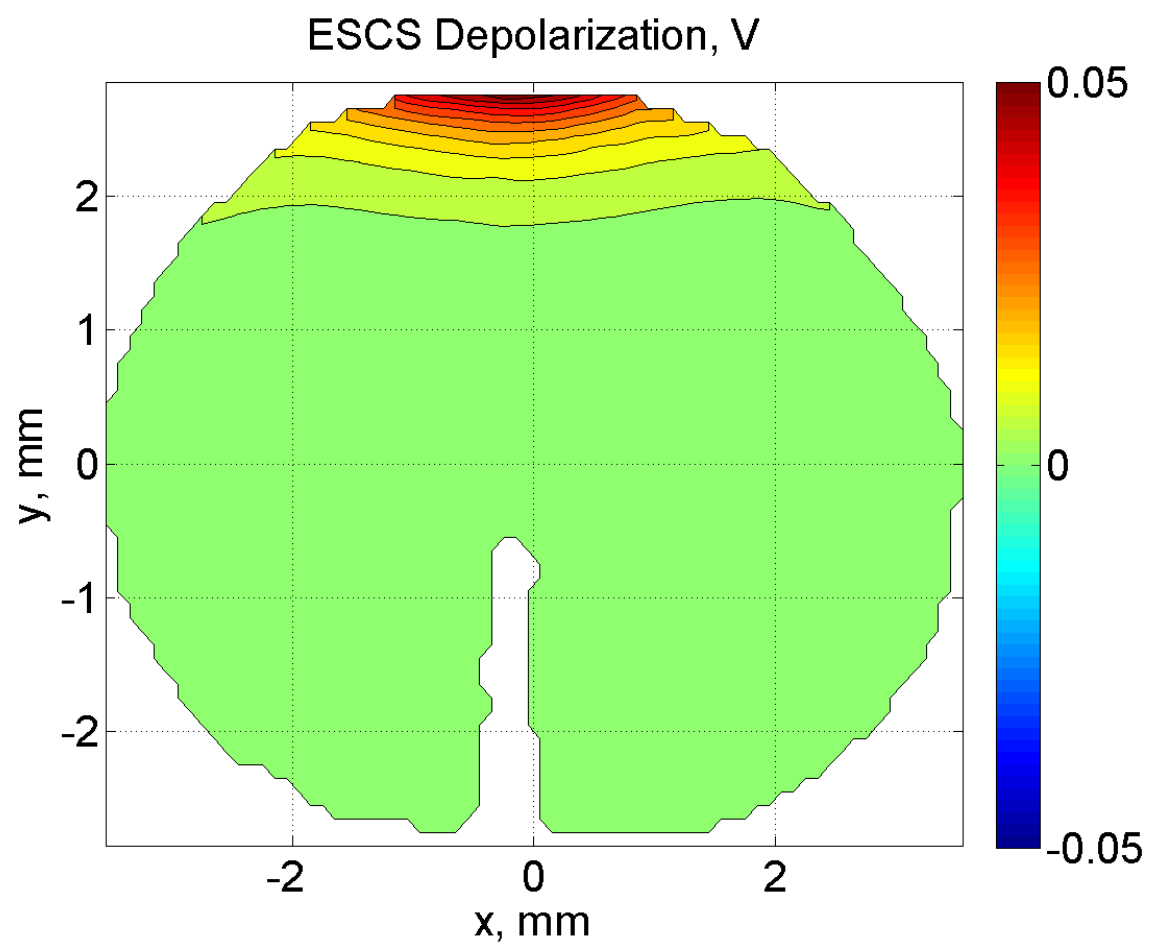

(c) ISCS

Figure 4.8: The depolarization voltage on cross sections at (a) $0.5 \mathrm{~mm}$ above cathode (b) on the same level at the cathode (c) $0.5 \mathrm{~mm}$ below the cathode; the color represents the amplitude of voltage in units of volts. 


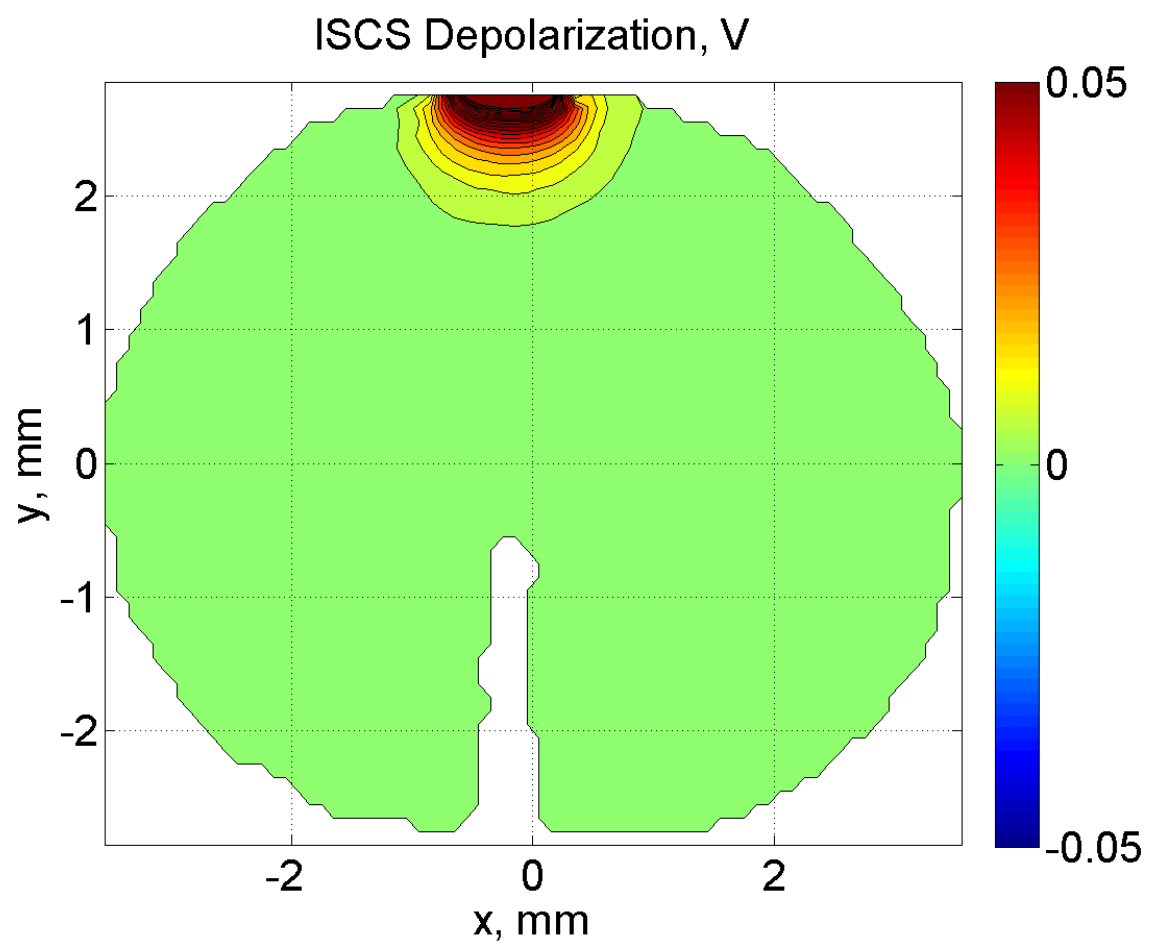

(a) ESCS

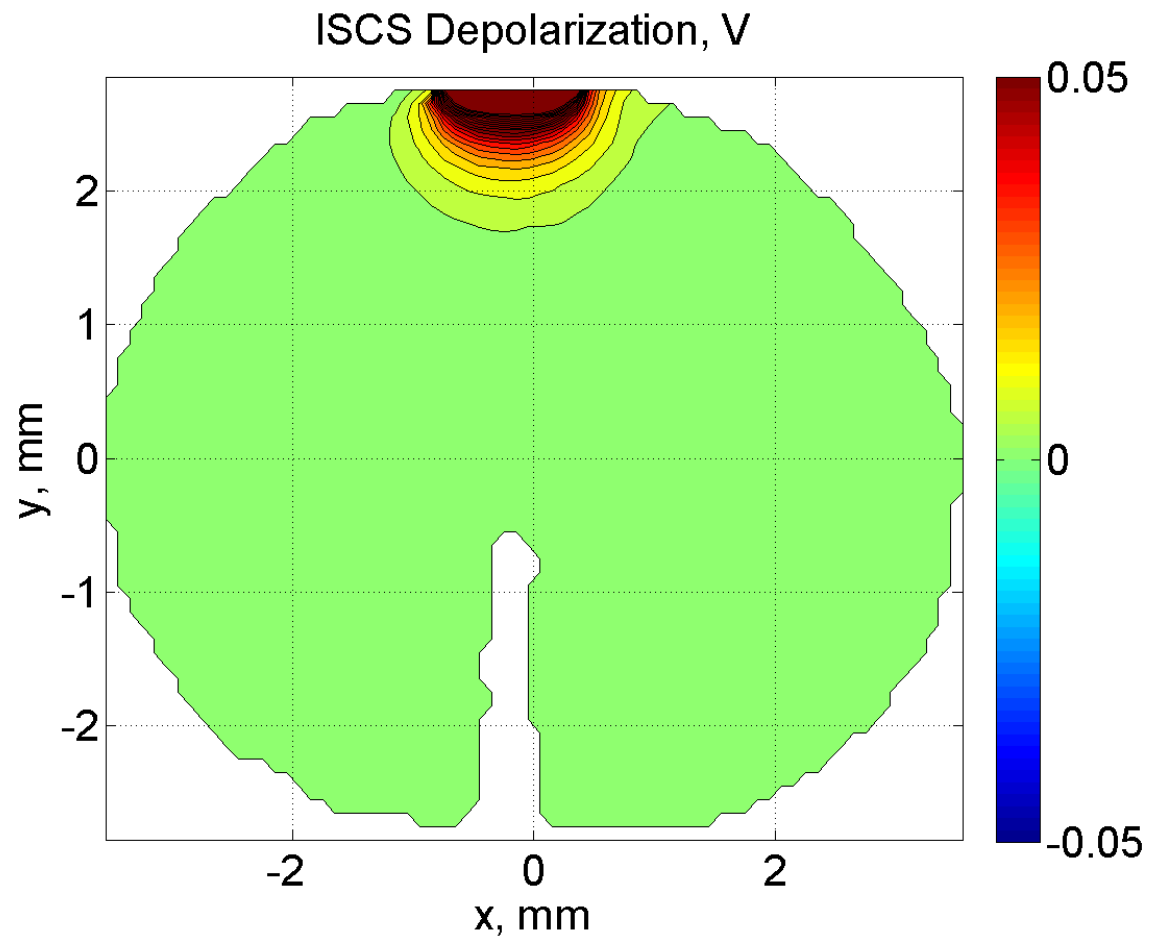

(b) ISCS

Figure 4.9: Continuing 


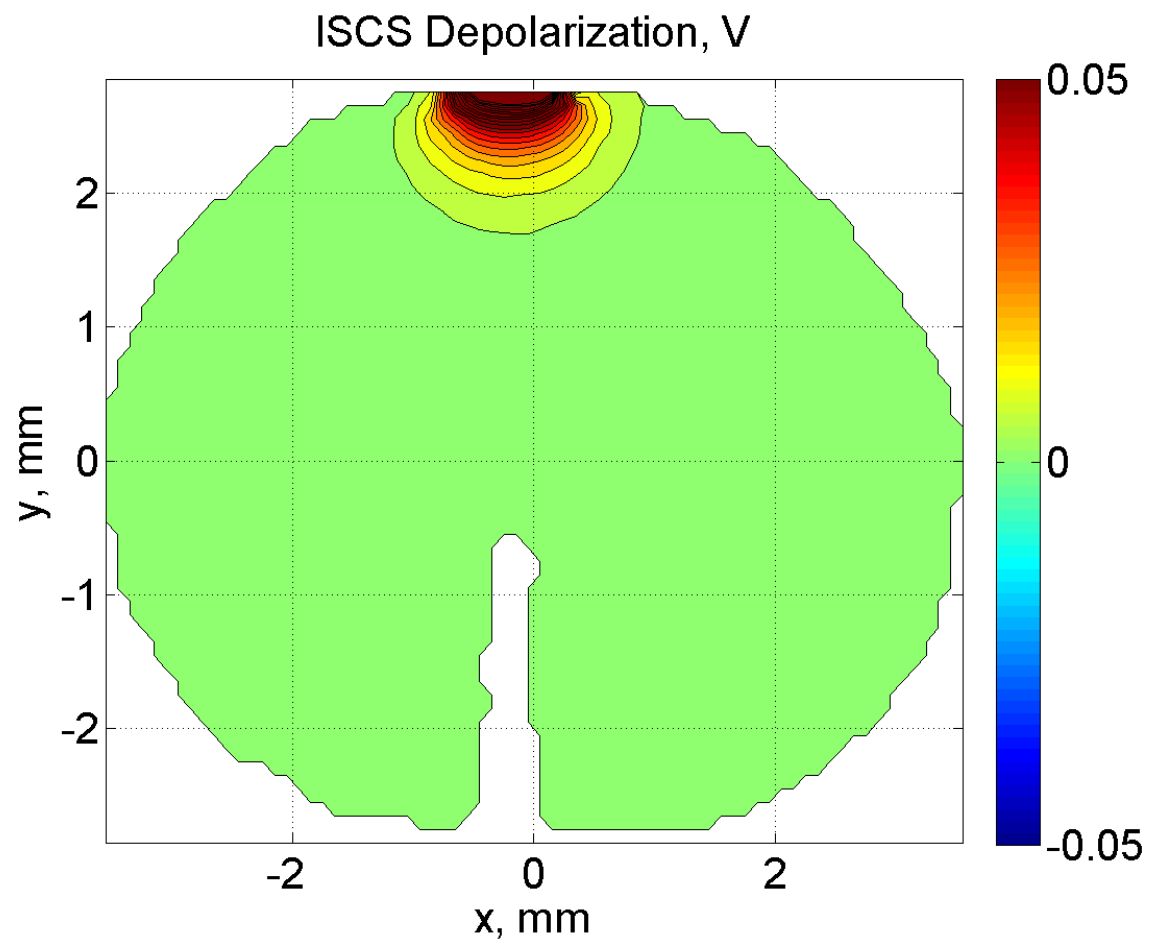

(c) ISCS

Figure 4.9: The depolarization voltage on cross sections (a) $0.5 \mathrm{~mm}$ above the cathode (b) on the same level as the cathode (c) $0.5 \mathrm{~mm}$ below the cathode; the color represents the amplitude of voltage in units of volts. 


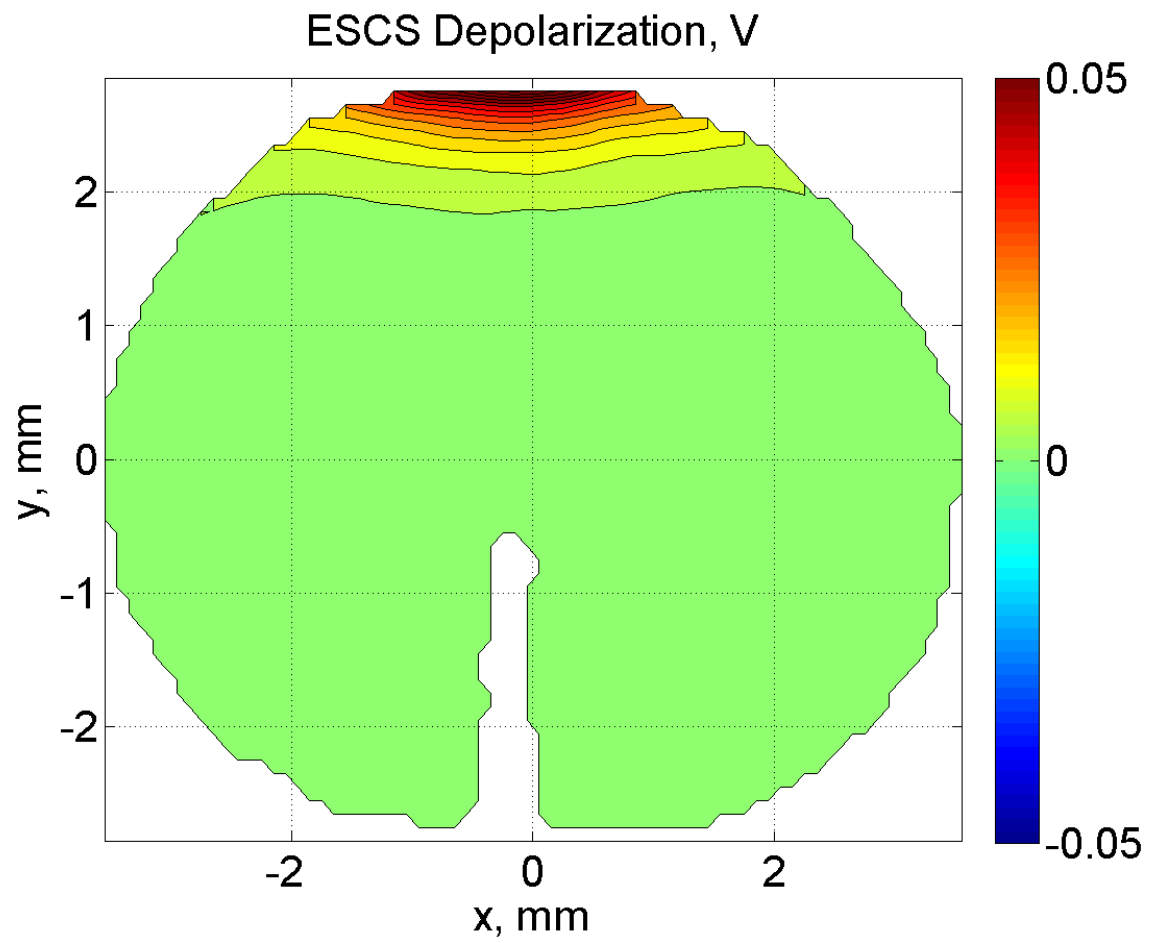

(a) ESCS

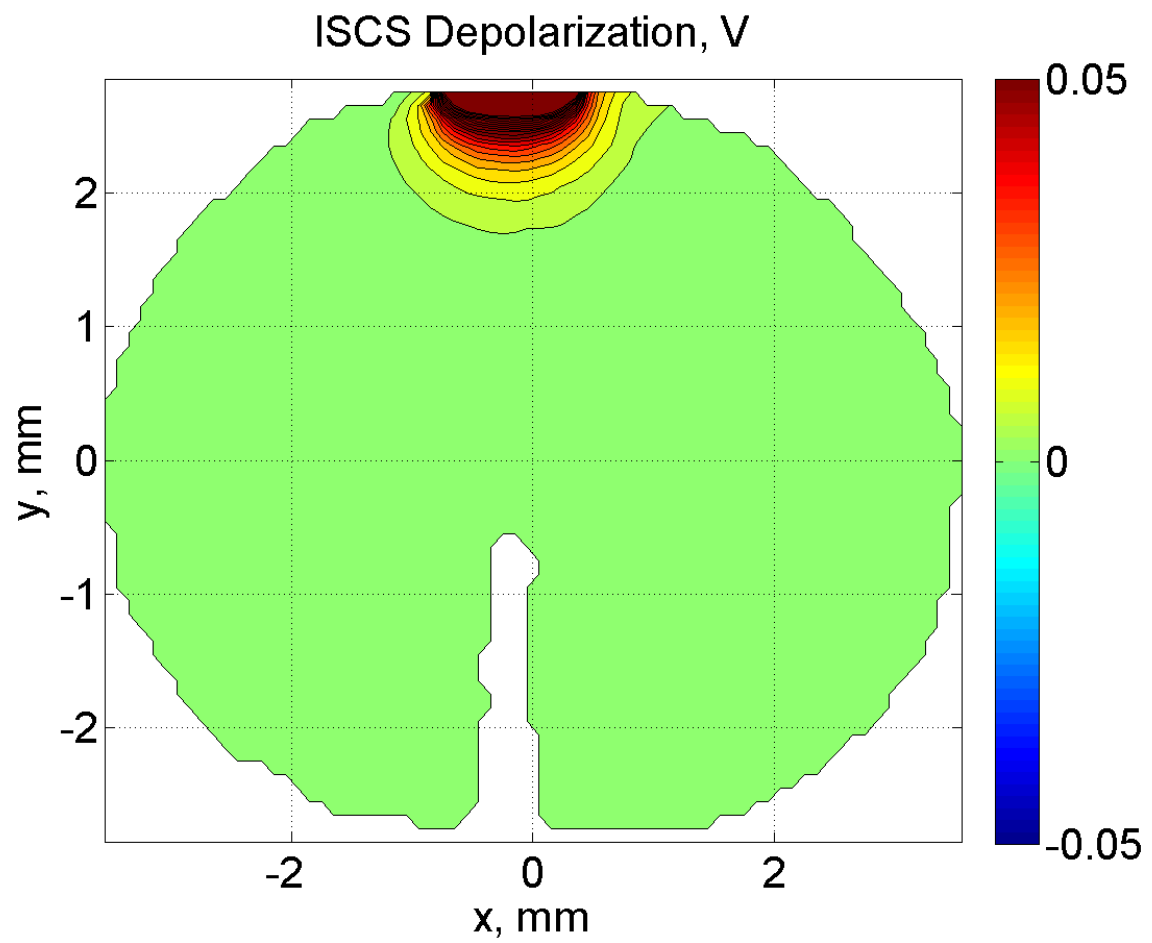

(b) ISCS

Figure 4.10: The depolarization voltage color contours of (a) ESCS and (b) ISCS; Cross sections parallel to the $X-Y$ plane are on the same level as the cathode. The legend shows the color code in the range between $-0.05 \mathrm{~V}$ and $0.05 \mathrm{~V}$. 


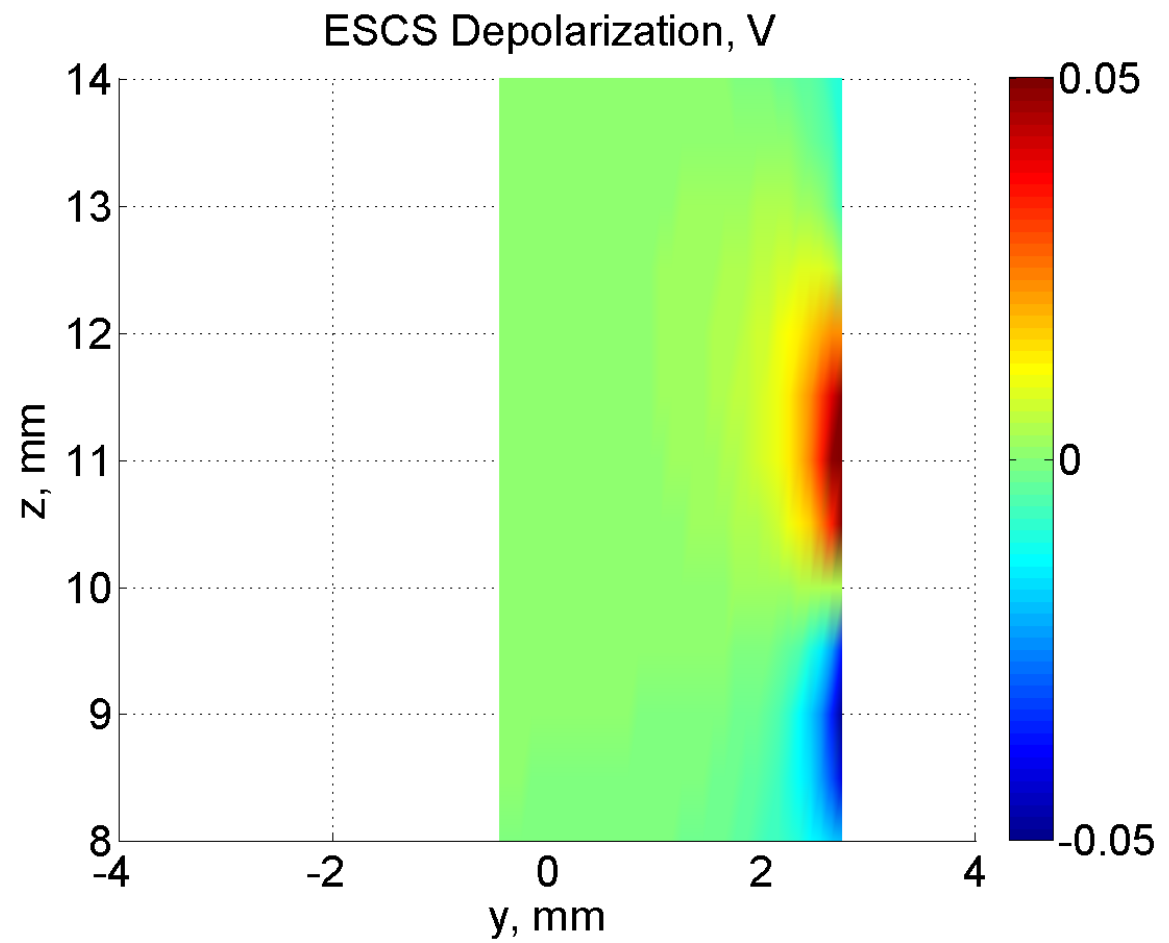

(a) ESCS

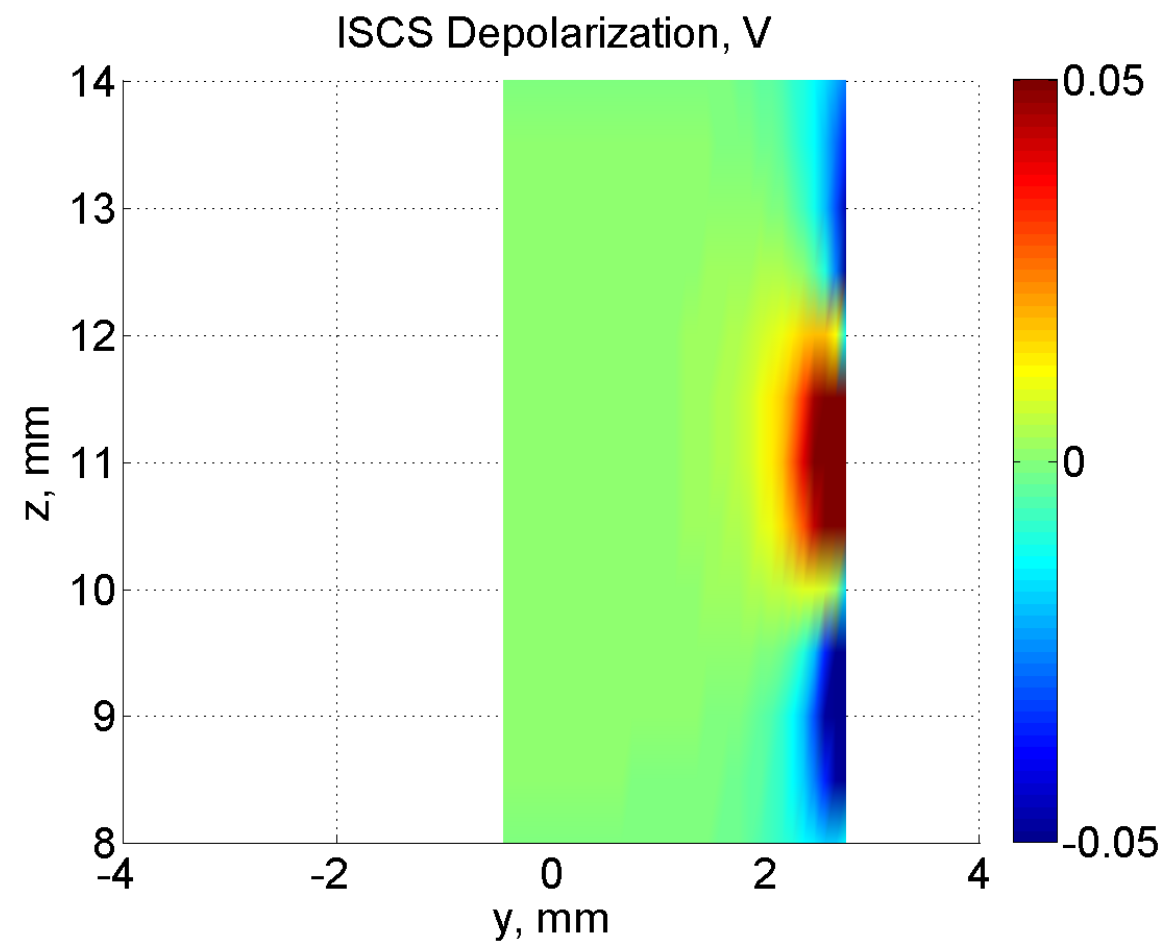

(b) ISCS

Figure 4.11: The depolarization voltage of (a) ESCS and (b) ISCS; the color represents the amplitude of voltage. Cross sections parallel to the $Y-Z$ plane are through the center of the spinal cord. 
The $Y-Z$ cross sections demonstrate that the depolarization voltages on nodes of the same fiber can have large differences. Any node on an individual fiber the reaches $25 \mathrm{mV}$ depolarization will initiate the potential transmission. The key nodes which change the excitation status of nerve fibers may spread at various vertical levels. But for vertically oriented DC fibers, the projection of key nodes on the horizontal plane shows a binary plot that distinguishes between excited and unexcited areas on the spinal cord (Fig. 4.12). The result illustrates that ISCS has better focus than ESCS, which is important in avoiding side effects. ESCS generates a thin and widespread excitation area covering the dorsal horn. Thus, this could lead to the undesired situation where dorsal root fibers in the dorsal horn are also excited. 


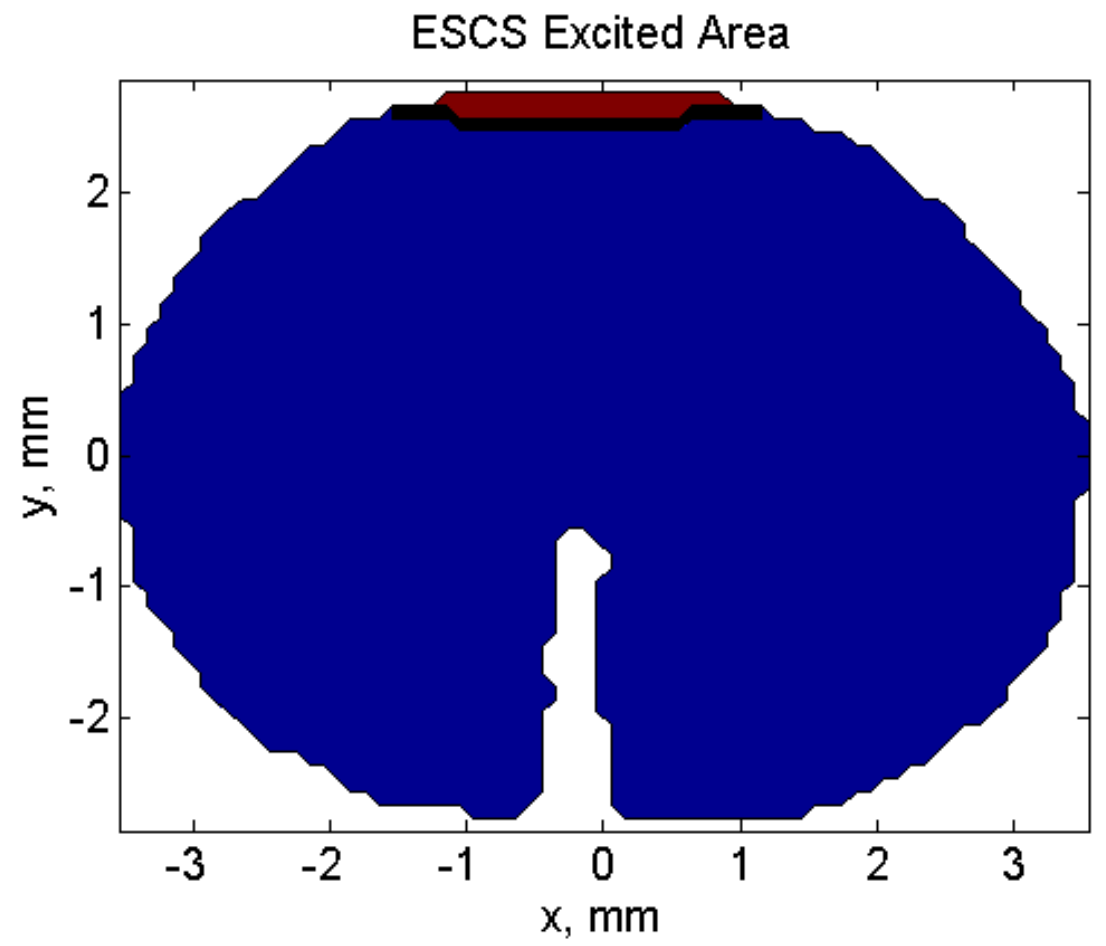

(a) ESCS

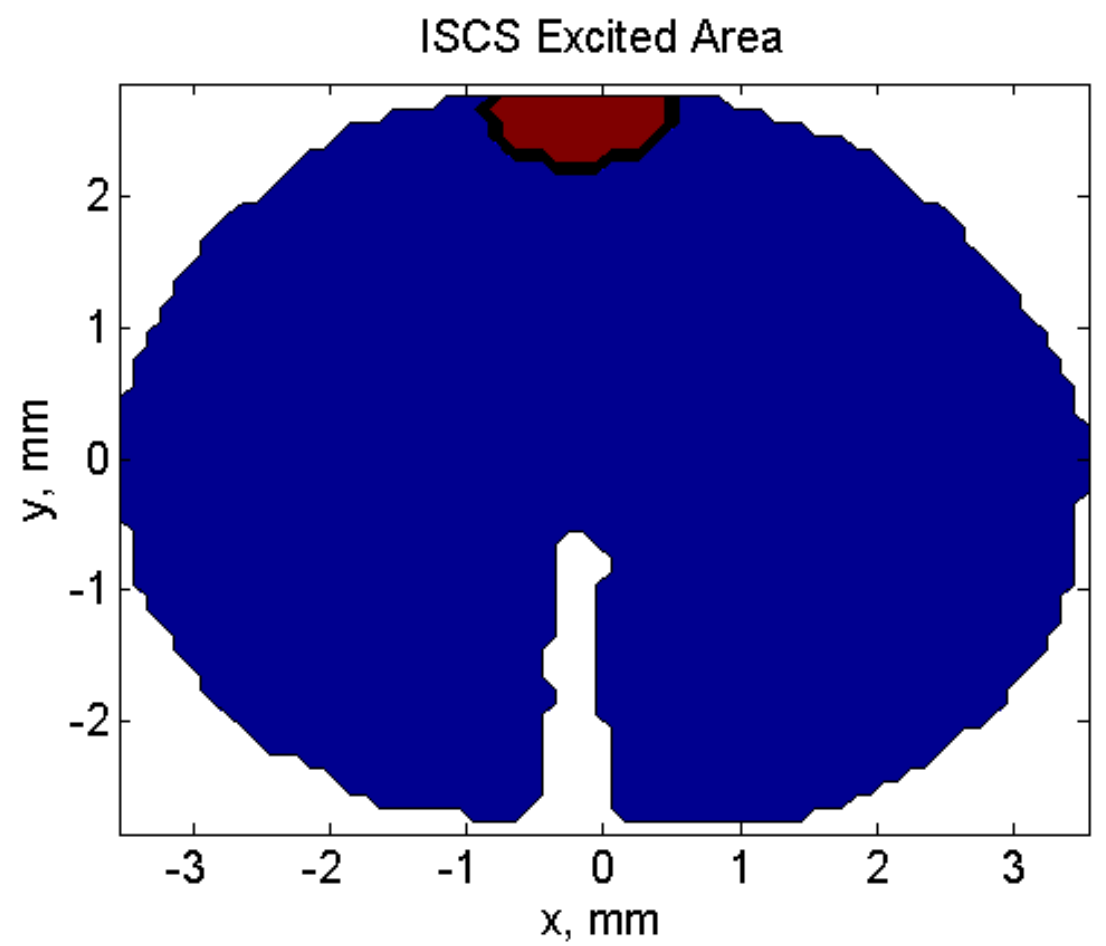

(b) ISCS

Figure 4.12: Red color shows excited area on the spinal cord under the condition of a $1 \mathrm{~V}$ applied voltage difference on the electrodes (a)ESCS has a wider and thinner excitation area than (b)ISCS. Because the Gracile fasciculus is located on the spinal cord from the medial to the lateral part, a focused excitation is essential to void side effects. 
Therefore, we can safely conclude that, regardless the type of stimulation source, ISCS always has a wider stimulation therapeutic window than ESCS. In other words, ISCS is able to fully stimulate target nerve fibers with a much smaller input on electrodes. Meanwhile, the shaper contours of ISCS allow the excitation area to focus on the targeted nerve fibers, diminishing the possibility of unwanted stimulations.

\subsection{Comparison between Modeling and Experimen- tal Results}

In past studies of ESCS, computational results are validated by statistical studies of clinical improvement after treatment. The lack of a directly comparison between modeling results and experimental results clouds understanding the SCS mechanism. Based on the 3-D depolarization result, we developed a new model which seeks explain the animal surgery results. 


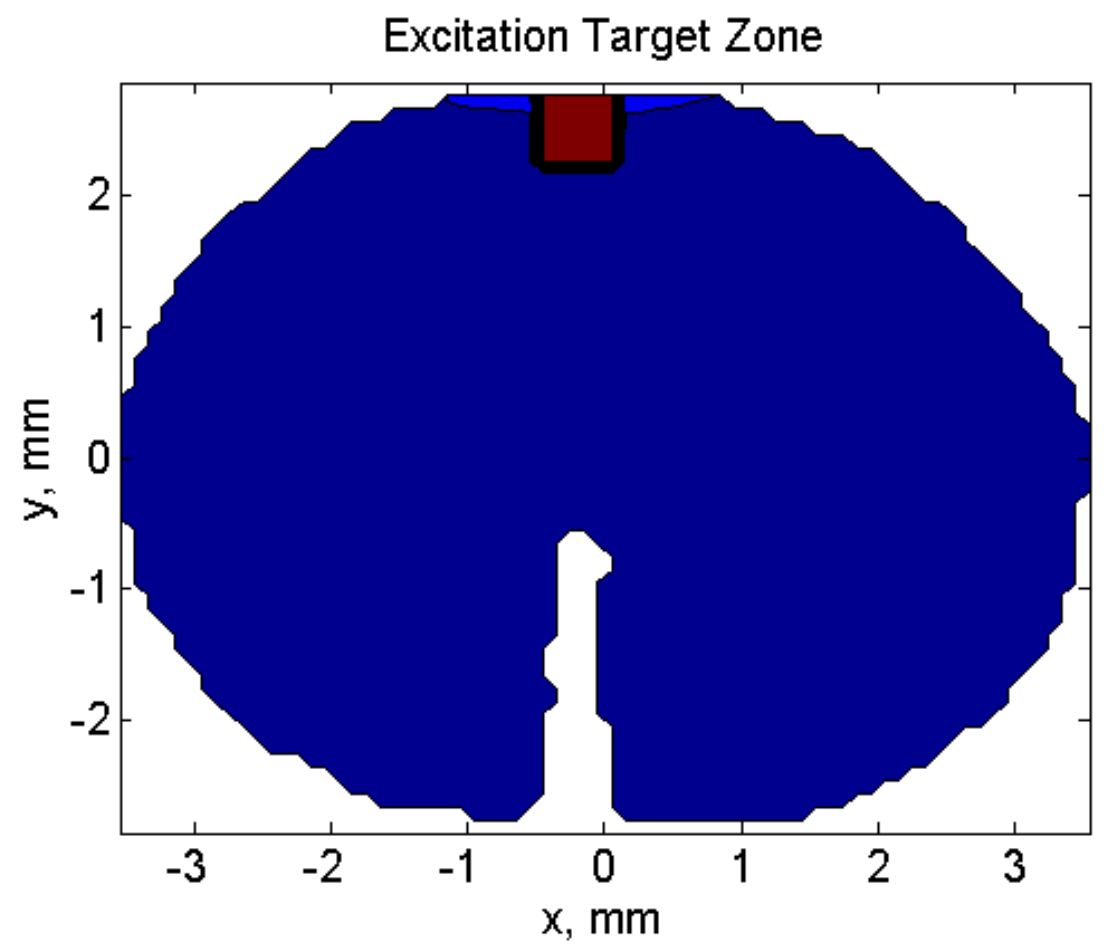

Figure 4.13: The blue area represents the horizontal cross section of the spinal cord; the red square is the excitation target with $0.5 \mathrm{~mm}$ side length. 
In the experiment mentioned above, the channel on the sheep brain with maximum evoked response potential (ERP) corresponded to a certain sensory zone stimulated on the spinal cord. In modeling, the target stimulation area is illustrated in Fig. 4.13 in red. We hypothesized that both the number of excited fibers in the excitation zone and the depolarization intensity are influential to ERP observation. If any node of a fiber has a depolarization voltage equal to or larger than $25 \mathrm{mV}$, the fiber is identified as excited. The saturation depolarization voltage is $100 \mathrm{mV}$ [35]. The maximum depolarization voltages of the excited DC fibers in the target area are summed. This value is compared with the ERPs with different excitation voltage (Fig. 4.14), which provides some evidence to support the hypothesis.

Data are fitted by exponential saturation curves:

Experimental result

$$
\begin{aligned}
& Y_{I S C S}=-411.612+\frac{1324.66}{1+2.218 e^{-5.62 x}} \\
& Y_{E S C S}=-10358.7+\frac{11191.7}{1+0.08 e^{-1.346 x}}
\end{aligned}
$$

Modeling result

$$
\begin{gathered}
Y_{I S C S}=-4.3799+\frac{7.9499}{1+0.8151 e^{-1.4834 x}} \\
Y_{E S C S}=-0.4258+\frac{3.6625}{1+7.6011 e^{-1.4828 x}}
\end{gathered}
$$




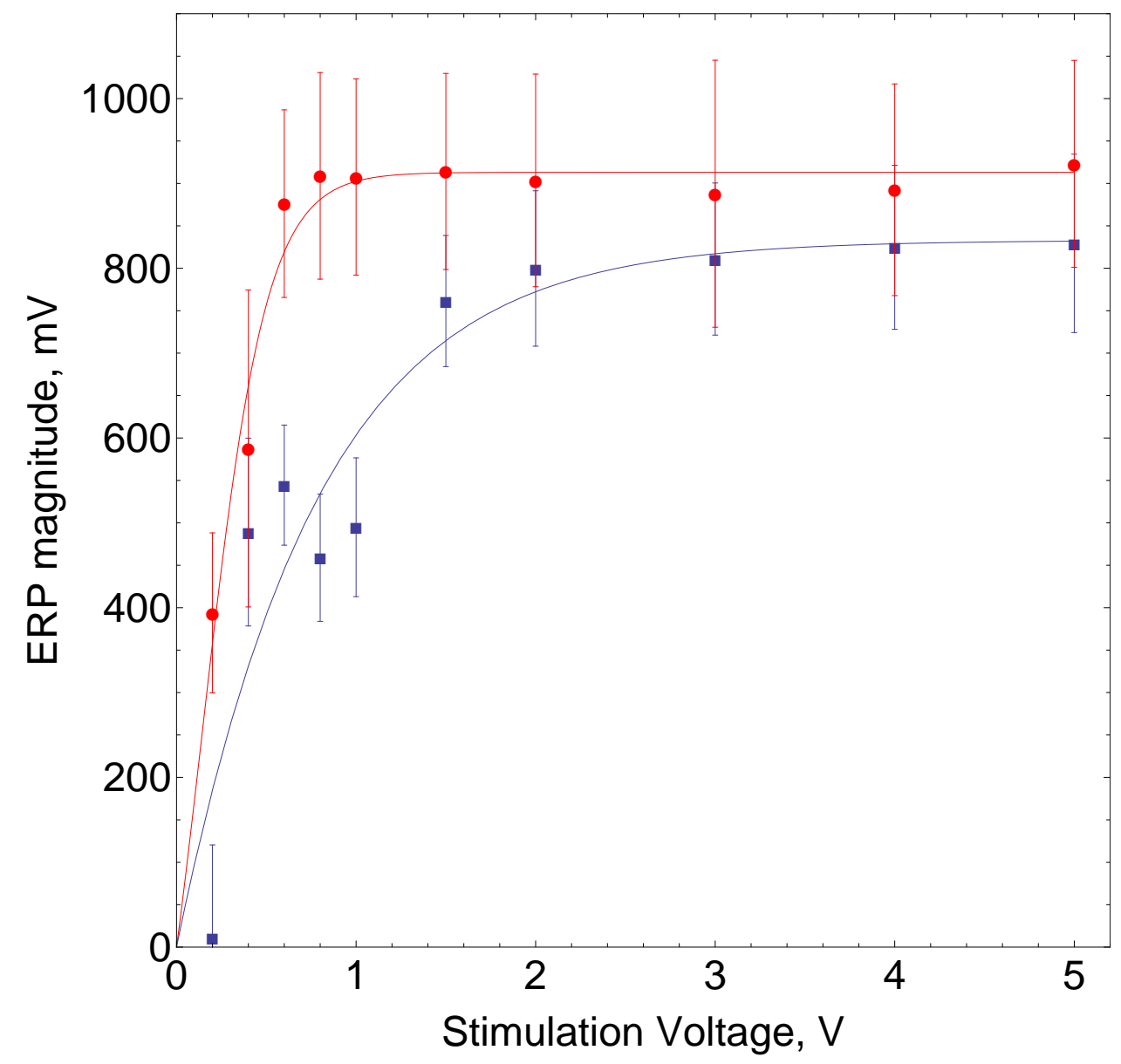

(a) Experimental result

Figure 4.14: Continuing 


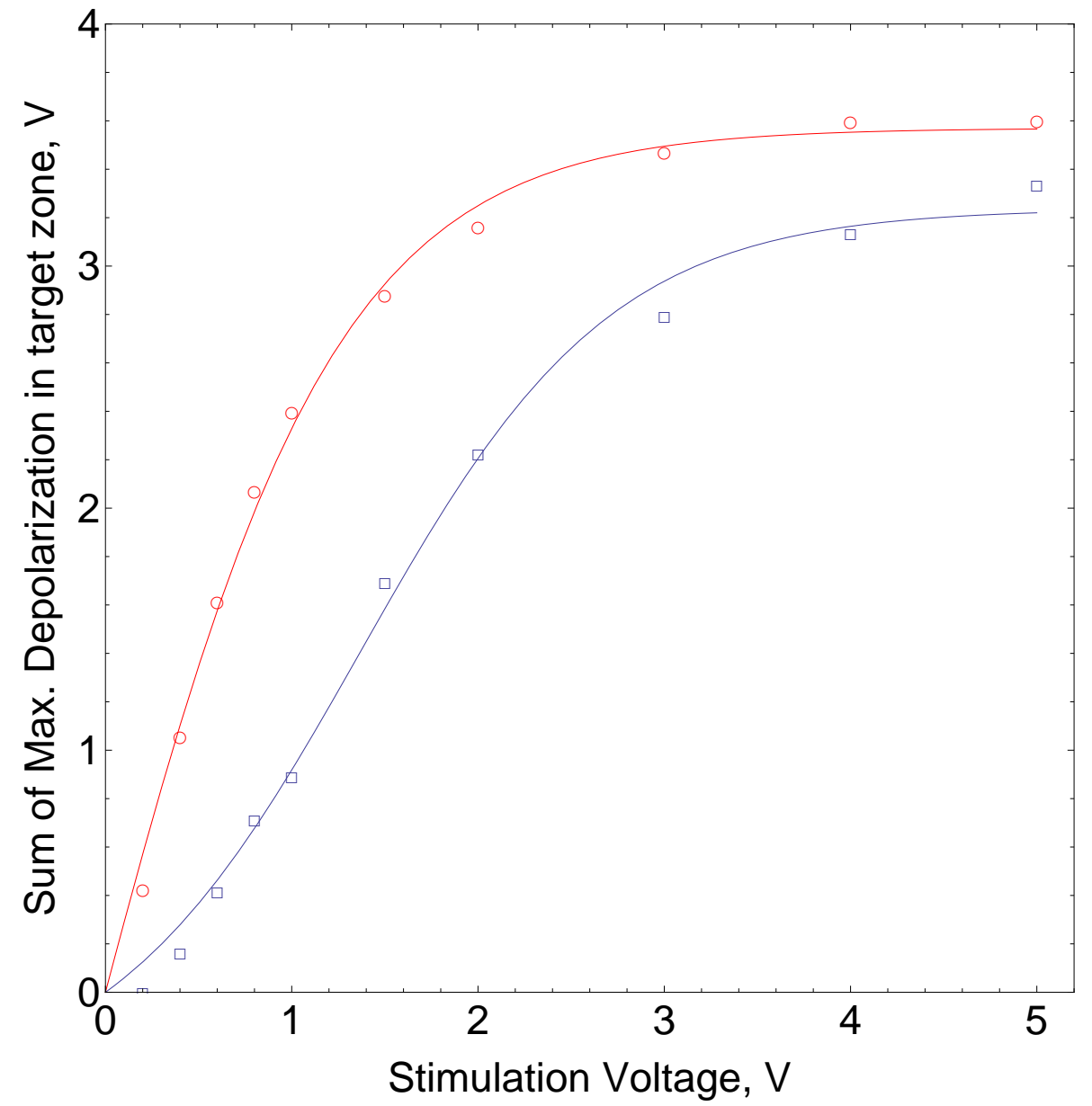

(b) Modelling result

Figure 4.14: With different applied voltages, the (a)experimental result shows the observed ERP at the brain channel with maximum response, and the associated fitted curves; while the (b) modelling result demonstrates the total maximum depolarization voltages of excited fibers in the target zone and the fitted curve. Red and blue colors here represent ISCS and ESCS respectively. 
As shown there, ISCS has a significantly larger response at low input voltages. For example, at $0 \mathrm{~mm}$ distance and at $0.2 \mathrm{~V}$ input, the intradural response is more than 10 times higher than the epidural response. This implies a substantially lower excitation threshold for the stimulation input. Moreover, ESCS and ISCS have different saturation amplitudes. The reason is that ISCS has higher maximum depolarization intensity for excited fibers in the target zone. A higher saturation amplitude is more promising to achieve consistent clinical treatment outcomes. Thirdly, in the range of increased phase, the slope of ISCS is about 2 times that of ESCS. The larger slope suggests more focused stimulation. In other words, more energy is conducted to the target neuron tissue and less perturbation caused to undesired tissues.

\subsection{Conclusion}

Both modelling and experimental results demonstrate that ISCS can largely decrease the input signal intensity threshold for exciting nerve fibers. The reason is that CSF has much higher electrical conductivity than any other tissue in the spinal cord. In epidural SCS, the CSF layer is like an electrical shield surrounding the spinal cord. Most of the electrical energy is conducted away by the CSF and is spread rather uniformly over a large area on the white matter. This effect impedes creating a high intensity, focused excitation spot on the white matter. ISCS virtually avoids this issue. When the electrodes are in contact with white matter, the input stimulation can directly influence nearby nerve fibers. ISCS can achieve a lower input stimulation threshold, a larger therapeutic window, and a smaller and more focused excitation

area. Those new effects associated with ISCS provide a solution to the effectiveness and safety dilemma encountered by ESCS.

SCS has been widely used in clinical application for more than forty years. How- 
ever, the therapeutic mechanism for it is still unclear. Becasue of the complexity of the nervous system, one of the main obstacles is to use modeling results to complete the connection between the excitation process in local nerve fibers and the perception of pain relief in the brain. Our theoretical model offers a step in that direction relative to the animal surgery result. Further validation of the theory needs controlled and long term studies involving a sufficient number of subjects. 


\section{Chapter 5}

\section{Future Scope and Directions}

\subsection{Overview}

In this work, an efficient computational model for ISCS has been developed and implemented. As a novel neural modulation method, ISCS is expected to offer a more effective pain treatment than ESCS. By using FEM and nerve fiber models, this theoretical study shows that ISCS has a much lower stimulation threshold and a much wider therapeutic window than ESCS. Thus, ISCS should be able to generate the required neural response with less stimulation input, while having less chance of causing side effects. Comparisons between ISCS and ESCS were conducted under the conditions of both current source stimulation and voltage source stimulation. Based on this computational model, a theory has been established to explain some of the experimental results of ISCS on animal subjects. It is a promising start to contribute on exploring the mechanism of SCS.

A review of studies of nerve stimulation and the evolution of ESCS has shown that the stimulation of a single nerve fibers has been well understood and ESCS has been widely applied with modest therapeutic effectiveness. However, very few have 
attempted to describe and explain the details of how the neuron system react to such external stimulation. Also, an inherent limitation of ESCS is that the shunting effect of CSF hinders this method from obtaining a balance between efficacy and safety. ISCS is designed to circumvent the issue by implanting electrodes in direct contact with the spinal cord white matter. Although the theory developed in this work attempted to understand SCS in terms of a cluster of fibers, and it agrees experimental results, further validation is clearly needed.

\section{$5.2 \quad$ Future Work}

Because the computational model is based on a short stimulation pulse which is much briefer than neural response time scale, results of depolarization voltages represent the initiation of an action potential. Considering the large number of fibers studied in this model, the transmission of action potential in a cluster of nerve fibers could be simulated with more advanced computational techniques.

Dorsal root fibers are critical paths for neural signal transmission in the nerve system. In development of ESCS, much work have been done to study the stimulation of dorsal root fibers. The excitation status of dorsal root fibers under the condition of ISCS should be investigated in in greater detail future studies.

The configuration of the electrodes has proven to be a significant parameter in the case of ESCS. Systematic studies should be conducted to determine the optimum stimulation efficacy among various electrodes configurations. This would be directly helpful for the clinical application of ISCS. 


\section{Bibliography}

[1] R. Melzak and P. D. Wall, "Pain mechanisms: a new theory," Science, vol. 150, pp. 971-979, 1965.

[2] C. N. Shealy, J. T. Mortimer, and J. B. Reswick, "Electrical inhibition of pain by stimulation of the dorsal columns: Preliminary clinical report," Anesthes. Analges.(Cleveland), vol. 46, pp. 489-491, 1967.

[3] L. Atkinson, S. R. Sundaraj, C. Brooker, J. OCallahan, P. Teddy, J. Salmon, T. Semple, and P. M. Majedi, "Recommendations for patient selection in spinal cord stimulation," J. Clin. Neurosci., vol. 18, pp. 1295-1302, 2011.

[4] K. Kumar, "Financial impact of spinal cord stimulation on the healthcare budget: a comparative analysis of costs in Canada and the United States," J. Neurosurg. Spine, vol. 10, pp. 564-573, 2009.

[5] C. C. de Vos, M. P. Hilgerink, H. P. J. Buschman, and J. Holsheimer, "Electrode contact configuration and energy consumption in spinal cord stimulation," Neursurgery, vol. 65, pp. 210-216, 2009.

[6] V. Sankarasubramanian, J. R. Buitenweg, J. Holsheimer, and P. Veltink, "Electrode alignment of transverse tripoles using a percutaneous triple-lead approach in spinal cord stimulation," Journal of Neural Engineering, vol.8, 016010, 2011. 
[7] J. Woo, C. A. Miller and P. J. Abbas, "The dependence of auditory nerve fiber rate adaption on electric stimulus parameters, electrode position and fiber diameter: a computer model study," JARO, vol. 11, pp. 283-296, 2010.

[8] E. N. Warman, W. M. Grill and D. Durand, "Modeling the Effects of Electric Fields on Nerve Fibers: Determination of Excitation Thresholds," IEEE Trans. Biomed. Eng., vol.39, pp. 1244-1254, 1992.

[9] W. A. Wesselink, and J. Holsheimer, "Electrical safety in spinal cord stimulation: current density analysis by computer modeling," In: IEEE 17th Annual Conference Engineering in Medicine and Biology Society, 20-23 Sept. 1995, Montreal, Canada.

[10] T. Cameron, "Safety and Efficacy of Spinal Cord Stimulation for the treatment of Chronic Pain: a 20-year Literature Review," J Neurosurg(Spine 3), vol.100, pp. 254-267, 2004.

[11] S. Eldabe, "An analysis of the components of pain, function, and health-related quality of life in patients with failed back surgery syndrome treated with spinal cord stimulation or conventional medical management," Neuromodulation, vol. 13, pp. 201-209, 2010.

[12] M. A. Howard III, M. Utz, T. J. Brennan, B. D. Dalm, S. Viljoen, J. K. Kanwal, and G. T. Gillies, "Biophysical attributes of an in vitro spinal cord surrogate for use in developing an intradural neuromodulation system," J. Appl. Phys., vol. 110, 04701, 2011.

[13] K. N. Gibson-Corley, H.Oya, O. E. Flouty, D. C. Fredericks, N. D. Jeffery, G. T. Gillies, and M. A. Howard III, "Ovine tests of a novel spinal cord neuromodulator and dentate ligament fixation method," J. Investig. Surg., in press. 
[14] O. E. Flouty, H. Oya, H. Kawasaki, S. Wilson, C. G. Reddy, N. D. Jeffery, T. J. Brennan, K. N. Gibson-Corley, M. Utz, G. T. Gillies, and M. A. Howard III, "A new device concept for directly modulating spinal cord pathways: initial in vivo experimental results," Physiol. Meas., vol. 33, pp. 2003-2015, 2012.

[15] O. E. Flouty, H. Oya, H. Kawasaki, C. G. Reddy, D. C. Fredericks, K. N. GibsonCorley, N. D. Jeffery, G. T. Gillies, and M. A. Howard III, "Intracranial somatosensory responses with direct spinal cord stimulation in anesthetized sheep," PLOS ONE, submitted for publication.

[16] A. L. Hodgkin and A. F. Huxley, "A Quantitative description of membrane current and its application to conduction and excitation in nerve," J. Physiol, vol. 117, pp. 500-544, 1952.

[17] B. Frankenhaeuser and A. F. Huxley, "The action potential in the myelinated nerve fiber of Xenopus Laevis as computed on the basis of voltage clamp data," J. Physiol, vol. 171, pp. 302-315, 1964.

[18] D. R. McNeal, "Analysis of a model for excitation of myelinated nerve," IEEE Trans. Biomed. Eng., vol. BME-23, pp. 329-337, 1976.

[19] J. D. Sweeney, D. Durand, and J. T. Mortimer, "A nerve cuff technical for selective excitation of peripheral nerve trunk regions," IEEE Trans. Biomed. Eng., vol. 37, pp. 706-715, 1990.

[20] F. Ratty, "Analysis of Models for External Stimulation of Axons," IEEE Trans. Biomed. Eng., vol. BME-33, pp. 974-977, 1986.

[21] F. Ratty, "Analysis of Models for Extracellular Fiber Stimulation," IEEE Trans. Biomed. Eng., vol. 36, pp. 676-682, 1989. 
[22] K. Kumar, C. Toth and R. Nath, et al "Epidural Spinal Cord Stimulation for treatment of Chronic Pain-some Predictors of Success. A 15-year Experience," Surg. Neurol., vol. 50, pp. 110-121, 1998.

[23] Waltz JM, Reynolds Lo and Riklan M, "Multi-lead spinal cord stimulation for control of motor disorders," Appl. Neurophysiol., vol. 44(4), p. 244-57, 1981.

[24] R. Fuentes, P. Petersson, W. B. Siesser et al, "Spinal Cord Stimulation Restores Locomotion in Animal Models of Parkinson's Disease," Science, vol. 323(5921), pp. 1578-1582, 2009.

[25] B. Coburn, "A theoretical study of epidural stimulation of the spinal cordPart II: Effect on long myelinated fibers," IEEE Trans. Biomed. Eng., vol. BME-32, pp. 978-986, 1985.

[26] J. J. Struijk, J. Holsheimer, and B. K. Veen van, "Analysis of dorsal column stimulation," In: 10th Annual International Conference of the IEEE Engineering in Medicine and Biology Society, 4-7 Nov. 1988, New Orleans, Louisiana.

[27] J. J. Struijk, J. Holsheimer, and B. K. Veen van, "Epidural Spinal Cord Stimulation: Calculation of Field Potentials with Special Reference to Dorsal Column Nerve Fibers," IEEE Trans. Biomed. Eng., vol.38, pp. 104-110, 1991.

[28] J. J. Struijk, J. Holsheimer, and H. B. K. Boom, "Excitation of dorsal root fibers in spinal cord stimulation: A theoretical study," IEEE Trans. Biomed. Eng., vol.40, pp. 632-639, 1993.

[29] J. Holsheimer, J. J. Struijk, "Electrode geometry and preferential stimulation of spinal nerve fibers having different orientations: a modeling study," In: 14th Annual International Conference of the IEEE Engineering in Medicine and Biology Society, Oct. 29 - Nov. 1 1992, Paris, France. 
[30] J. Holsheimer, W. A. Wesselink, "Optimum electrode geometry for spinal cord stimulation: The narrow bipole and tripole," Med. Biol. Eng. Comput., vol.35, pp. 493-497, 1997.

[31] B. Coburn, "Electrical stimulation of the spinal cord: Two-dimensional finite element analysis with particular reference to epidural electrodes," Med. Biol. Eng. Comp., vol. 18, pp. 573-584, 1980.

[32] Comsol Multiphysics Reference Guide, Version 4.2a, COMSOL Inc., Burlington, MA, 2011.

[33] J. C. Oakley and J. P. Prager, "Spinal cord stimulation: Mechanisms of Action," SPINE, vol. 27, pp. 2574-2583, 2002.

[34] R. A. Serway, "Principles of Physics," 2nd ed. Fort Worth, Texas; London: Saunder College Pub. 1998. p. 602.

[35] A. Ferroni and D. Blanchi, "Maximum Rate of Depolarization of Single Muscle Fiber in Normal and Low Sodium Solutions," The Journal of General Physiology, vol. 49, pp. 17-25, 1965. 